\title{
Study on Roll Instability Mechanism and Stability Index of Articulated Steering Vehicles
}

\author{
Xuefei Li, Ya Wu, Wei Zhou, and Zongwei Yao \\ College of Mechanical Science and Engineering, Jilin University, Changchun 130022, China \\ Correspondence should be addressed to Zongwei Yao; yaozongwei@163.com
}

Received 2 November 2015; Accepted 1 March 2016

Academic Editor: Yuri Vladimirovich Mikhlin

Copyright (C) 2016 Xuefei Li et al. This is an open access article distributed under the Creative Commons Attribution License, which permits unrestricted use, distribution, and reproduction in any medium, provided the original work is properly cited.

\begin{abstract}
This study examines the roll instability mechanism and stability index of articulated steering vehicles (ASVs) by taking wheel loaders as the research object. A seven-degree-of-freedom nonlinear dynamics model of the ASVs is built on the basis of multibody dynamics. A physical prototype model of an ASV is designed and manufactured to validate the dynamic model. Test results reasonably agree with the simulation results, which indicates that the established dynamic model can reasonably describe ASV movements. Detailed analysis of the rollover stability of the wheel loader is performed with the use of the established dynamic model. Analysis results show that rollover will occur when the roll angular velocity exceeds a critical threshold, which is affected by lateral acceleration and slope angle. On this basis, a dynamic stability index applicable to the ASVs is presented.
\end{abstract}

\section{Introduction}

Both maneuverability and traction of off-road vehicles are important for enhanced performance in applications on unpredictable and changing terrains. To combine these two important features, articulated frame steering has become popular, and an increasing number of manufacturers choose this simple but efficient steering system. Many wheel loaders, scrapers, and forestry skidders are articulated steering vehicles (ASVs).

Modern ASVs, such as wheel loaders, have two separate parts that are connected by a steering hinge. The wheel axles are unsuspended, such that the rear axle is mounted on a pivoting joint to enable wheels contact on uneven ground. A schematic diagram of a wheel loader is shown in Figure 1. This structure is limited by decreased stability when turning because the center of gravity (CG) is laterally displaced. In addition, ASVs are off-road vehicles that work on steep slopes, unexpected banks, or uneven terrain, which result in a high probability of rollover accident.

Many scholars have focused on the stability and dynamics of such vehicles as cars, heavy trucks, and tractors. However, limited work has been conducted on the stability and handling performance of the ASVs, especially in terms of their motions on slopes and uneven terrain. Azad et al. [1] built a linear ASV model that can be used to analyze snaking instability. Yavin [2] built a simple dynamical model of the ASV on a horizontal plane. The vertical movement and the relative movement between the rear body and the rear axle were not considered in the model, such that it cannot be used to analyze instability when the ASVs pass over obstacles. Corke and Ridley [3] and Dragt et al. [4] studied the steering kinematics of the ASVs on the basis of plane models that do not consider vertical movement.

To evaluate vehicle stability quantitatively, many stability indexes have previously been developed for given conditions or certain prototype vehicles. Some existing stability indexes [5-7] are only based on acceleration or roll angular velocity threshold values. Given that the ASVs are off-road vehicles that travel on complex roads and work under poor conditions, vehicle roll stability cannot be accurately reflected if only a single lateral acceleration or roll angular velocity is used as a stability index. For example, instant lateral acceleration of ASV is large when passing over obstacles, but the vehicle does not lose stability in this case. As another example, the small roll angular speed of ASVs may cause instability when a vehicle is traveling on sloping road.

Efforts have been exerted to predict vehicle rollover on the basis of vehicle roll energy and tip-over potential energy. 


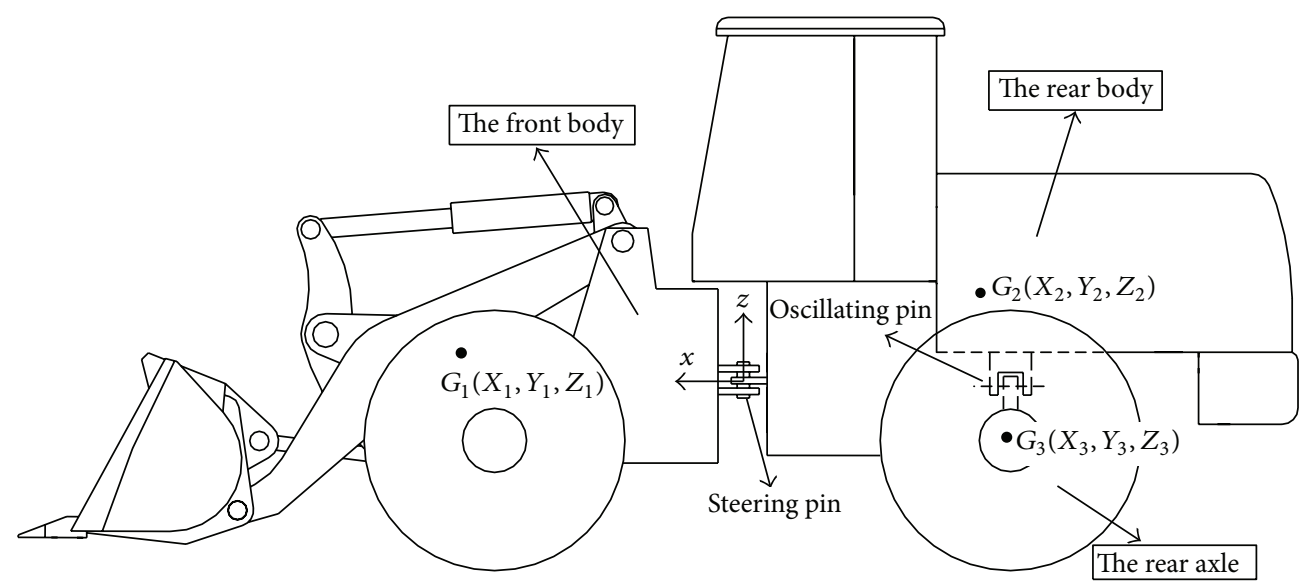

(a)

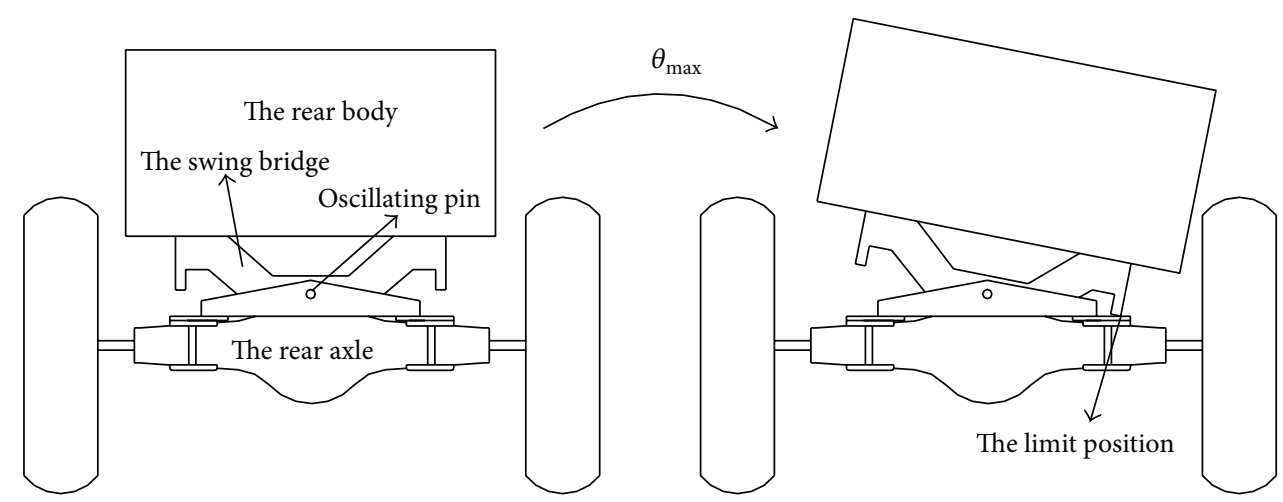

(b)

FIGURE 1: The structure outline of a wheel loader.

Nalecz et al. [8] suggested an energy-based function called the rollover prevention energy reserve (RPER). PRER is defined as the difference between the energy needed to bring the vehicle to its tip-over position and the rotational kinetic energy, which can be transformed into gravitational potential energy to lift the vehicle. Given the relative rotation between the front and rear bodies of the ASVs, the moment of inertia and CG position of the vehicle are changing in real time, such that the PREP of the ASVs is difficult to calculate.

Liu and Ayers $[9,10]$ proposed a dynamic stability index composed of lateral angle and lateral angular velocity. This index does not consider the effect of lateral acceleration on the ASVs instability. In fact, the ASVs are likely to lose stability because of large lateral acceleration when turning.

Preston-Thomas and Woodrooffe [11] used the lateral load transfer ratio (LTR) to indicate rollover threat. LTR can vary from 0 when the loads carried by the tires are equal to 1 when the tires lift off the road. In the calculation of LTR, the contact pressure of each tire must be known. However, tire contact pressure is difficult to measure. To avoid the direct measurement of tire contact pressure, some scholars approximately calculate LTR on the basis of the suspension system [12-14]. However, currently available ASVs have no suspension system.

By summarizing existing research, it can be found that the dynamic models of the ASVs were built without considering the impact of nonstructural terrain. In fact, sudden change in terrain is one of the main causes of instability. The existing models do not consider the impact of the swing bridge on the roll stability of the ASVs. In addition, the existing stability indexes are not entirely suitable for the ASVs.

Therefore, the physical model and stability index for the ASVs still need improvement. This research aims to build a nonlinear dynamic model that considers articulated steering movement, swing bridge movement, and ground environmental parameters to obtain the instability mechanism and dynamic rollover stability index for the ASVs.

\section{Modelling of the ASVs}

The typical ASVs comprise three parts, namely, the front body, the rear body, and the rear axle, as shown in Figure 1. Each part has three translational DOFs and three revolute DOFs, but all 18 DOFs are not completely independent. The longitudinal movements, the lateral movements, the vertical movements, and the pitch movements of the front body, the rear body, and the rear axle are interrelated. The roll motions of the front body and the rear body are also interrelated. The yaw movements of the rear body and the rear axle are related too. Meanwhile, the yaw movement of the front body is also related to that of the rear body. When the vehicle starts turning, the yaw rate difference between the front body and 


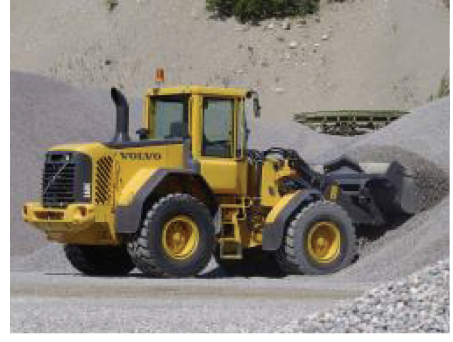

(a) Loading

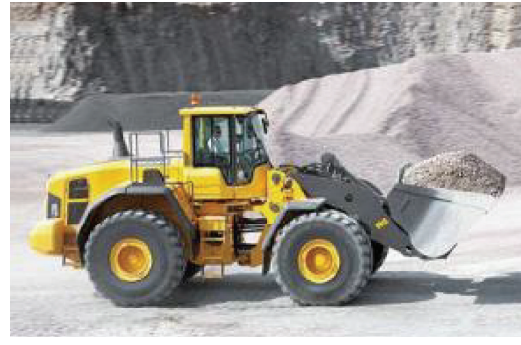

(b) Transporting

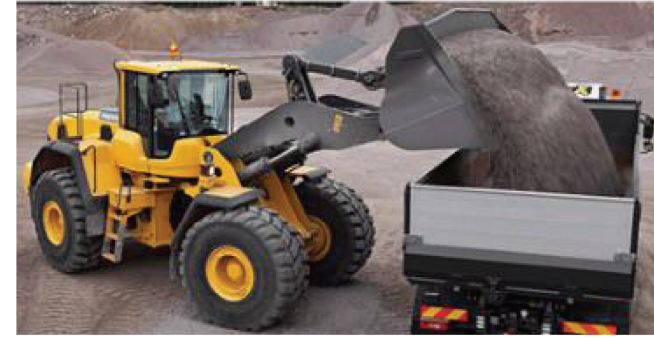

(c) Discharging

FIgURE 2: A typical operation process of wheel loader.
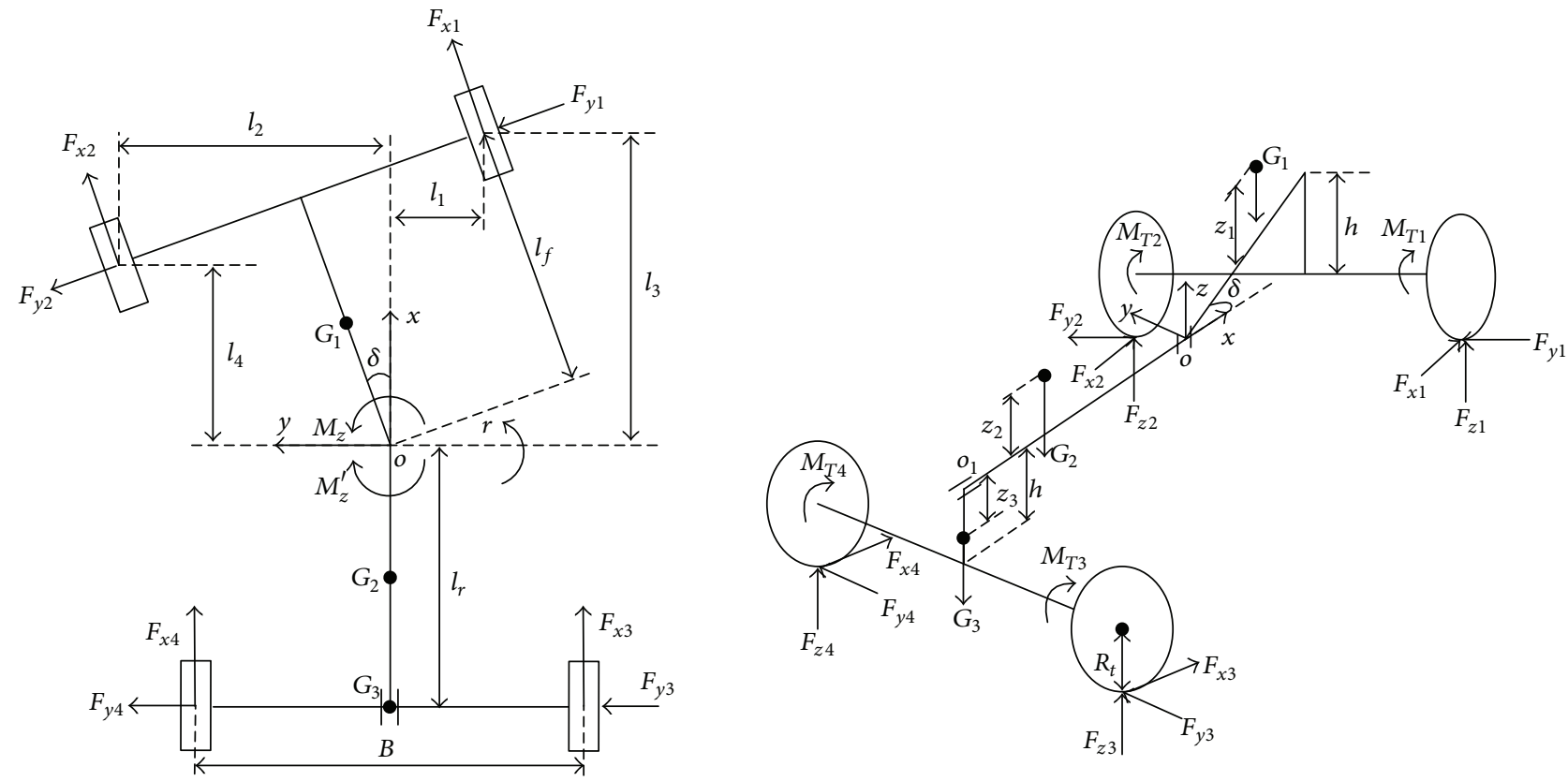

FIgURE 3: The model outline of vehicle movements.

the rear body is equal to the speed value of the steering angle. When the steering input is finished, the yaw rate of the front body is equal to that of the rear body.

According to the structure characteristics of the swing bridge, the roll motion of the ASVs includes two phases.

Instability of Level 1. The swing bridge does not reach the limit position. The rear body and the rear axle roll over around the oscillating pin. There are relative movements between the two.

Instability of Level 2. The swing bridge reaches the limit position and comes into contact with the rear axle. There are no further relative movements between the rear axle and the rear body.

In addition, the ASVs, such as the wheel loaders, are the heavy mining and construction equipment, which function in loading, transporting, and discharging materials as displayed in Figure 2. Load weight and arm position are changing when the ASVs are loading and discharging materials, which have an impact on the stability of the ASVs. However, the forward speed is very slow or even stops when the ASVs are loading and discharging materials, as shown in Figures 2 (a) and 2(c), and the risk of rollover is very low in these conditions. In fact, the ASVs are more likely to lose stability in the transporting state, as shown in Figure 2(b), and, in general, load weight and arm position remain unchanged in the transporting state. In order to simplify the analysis, this paper just considers the stability of the ASVs in the transporting state; that is, the load weight and arm position are assumed to be changeless.

Through the analysis, it is found that the following seven degrees of freedom can describe the movements of the ASVs: (1) the longitudinal velocity of the vehicle $v_{x}$; (2) the lateral velocity of the vehicle $v_{y}$; (3) the vertical movement of the vehicle $z$; (4) the pitch movement of the vehicle $\psi$; (5) the roll movement of the front body and the rear body $\theta$; (6) the roll movement of the rear axle $\theta^{\prime}$; (7) the yaw movements of the front body, the rear body, and the rear axle $r$.

Figure 3 shows the movements, the structure dimensions of the ASVs, and the forces on tires. The reference point of the coordinate system of the vehicle coincides with 
the intersection point of the articulated steering pin axis and the oscillating pin axis, which is connected with the rear body. The $x$-axis is parallel to the chassis plane of the rear body. It points to the forward direction of the perpendicular bisector of the rear axle. The positive direction of the $z$-axis is perpendicular to the horizontal plane. The positive direction of the $y$-axis is determined by the right-hand rule.

According to D'Alembert principle, the dynamics equations of the ASVs are written as follows:

(1) Vehicle motion along the $x$-axis direction:

$$
\begin{aligned}
& m\left(\dot{v}_{x}-v_{y} r+\dot{z} \dot{\psi}\right) \\
& =\sum_{i=1}^{2}\left[\left(F_{x i}+\frac{M_{T i}}{R_{t}}\right) \cos \delta-F_{y i} \sin \delta\right] \\
& \quad+\sum_{i=3}^{4}\left(F_{x i}+\frac{M_{T i}}{R_{t}}\right) .
\end{aligned}
$$

(2) Vehicle motion along the $y$-axis direction:

$$
\begin{aligned}
& m\left(\dot{v}_{y}+v_{x} r-\dot{z} \dot{\theta}\right) \\
& =\sum_{i=1}^{2}\left[\left(F_{x i}+\frac{M_{T i}}{R_{t}}\right) \sin \delta+F_{y i} \cos \delta\right]+\sum_{i=3}^{4} F_{y i} .
\end{aligned}
$$

(3) Vehicle motion along the $z$-axis direction:

$$
m\left(\ddot{z}+v_{y} \dot{\theta}-v_{x} \dot{\psi}\right)=\sum_{i=1}^{4} F_{z i}-\sum_{i=1}^{3} G_{i} \cos \theta_{i} \cos \psi,
$$

where $\theta_{1}=\theta_{2}=\theta, \theta_{3}=\theta^{\prime}$.

(4) The roll motion of the front body and the rear body:

$$
\begin{aligned}
\left(J_{x x 1}\right. & \left.+J_{x x 2}\right) \ddot{\theta} \\
= & \sum_{i=1}^{2}\left[\left(F_{x i}+\frac{M_{T i}}{R_{t}}\right) \sin \delta+F_{y i} \cos \delta\right]\left(R_{t}+h\right) \\
& \quad-F_{z 1} l_{1}+F_{z 2} l_{2}-G_{1}\left(X_{1} \sin \delta-Z_{1} \sin \theta\right) \\
& +G_{2} Z_{2} \sin \theta+\sum_{i=1}^{2} m_{i} a_{n i} Z_{i} \cos \theta-F_{S} B_{1} .
\end{aligned}
$$

(5) The roll motion of the rear axle:

$$
\begin{aligned}
J_{x x 3} \ddot{\theta}^{\prime}= & \left(F_{y 3}+F_{y 4}\right)\left(R_{t}+h\right)+\frac{\left(F_{z 4}-F_{z 3}\right) B}{2} \\
& +G_{3} Z_{3} \sin \theta^{\prime}+m_{3} a_{n 3} Z_{3} \cos \theta^{\prime}+F_{S} B_{2} .
\end{aligned}
$$

(6) Vehicle pitch motion around the $y$-axis:

$$
\begin{aligned}
& \left(J_{y y 1}+J_{y y 2}+J_{y y 3}\right) \ddot{\psi}=-F_{z 1} l_{3}-F_{z 2} l_{4}+\left(F_{z 3}+F_{z 4}\right) l_{r} \\
& +G_{1} X_{1} \cos \delta+G_{2} X_{2}-G_{3} l_{r}+\sum_{i=1}^{3} m_{i} \dot{v}_{x} Z_{i} \\
& +\left\{\sum_{i=1}^{2}\left[F_{y i} \sin \delta-\left(F_{x i}+\frac{M_{T i}}{R_{t}}\right) \cos \delta\right]\right. \\
& \left.-\sum_{i=3}^{4}\left(F_{x i}+\frac{M_{T i}}{R_{t}}\right)\right\}\left(R_{t}+h\right) .
\end{aligned}
$$

(7) Yaw motion of the rear body and the rear axle:

$$
\left(J_{z z 2}+J_{z z 3}\right) \dot{r}=-\left(F_{y 3}+F_{y 4}\right) l_{r}+\sum_{i=2}^{3} m_{i} a_{n i} X_{i}-M_{z}^{\prime} .
$$

(8) Yaw motion of the front body:

$$
J_{z z 1}(\dot{r}+\ddot{\delta})=\left(F_{y 1}+F_{y 2}\right) l_{f}-m_{1} a_{n 1} X_{1}+M_{z},
$$

where

$$
\begin{aligned}
l_{1} & =\frac{B}{2} \cos \delta-l_{f} \sin \delta, \\
l_{2} & =\frac{B}{2} \cos \delta+l_{f} \sin \delta, \\
l_{3} & =l_{F} \cos \delta+\frac{B}{2} \sin \delta, \\
l_{4} & =l_{F} \cos \delta-\frac{B}{2} \sin \delta, \\
a_{n 1} & =\dot{v}_{y}+v_{x} r-\dot{z} \dot{\theta}, \\
a_{n 2} & =\dot{v}_{y}+v_{x} r-\dot{z} \dot{\theta}, \\
a_{n 3} & =\dot{v}_{y}+v_{x} r-\dot{z} \dot{\theta}^{\prime} .
\end{aligned}
$$

$F_{S}$ is the contact force which is generated when the swing bridge reaches the limit position. In order to calculate $F_{S}$, the contact process is assumed to be connected by a spring and damping system, as shown in Figure $4 ; F_{S}$ can be written as follows:

$$
F_{S}=K_{S}\left(B_{1} \theta-B_{2} \theta^{\prime}\right)+C_{S}\left(B_{1} \dot{\theta}-B_{2} \dot{\theta}^{\prime}\right),
$$

where $K_{S}$ is contact stiffness and $C_{S}$ is contact damping. $K_{S}=$ 0 and $C_{S}=0$ when the swing bridge does not reach the limit position. $B_{2}$ is the distance between the oscillating pin and the limit position, $B_{1} \approx B_{2}$.

\section{The Tire Model}

The Fiala tire model describes the relationship among the lateral force, the vertical force, the longitudinal force, the slip angle, and the slip ratio in detail, and, furthermore, it has been verified by experiments [15]. Therefore, the Fiala tire model is adopted to calculate the longitudinal force, the vertical force, and the lateral force of the tires in this paper. 


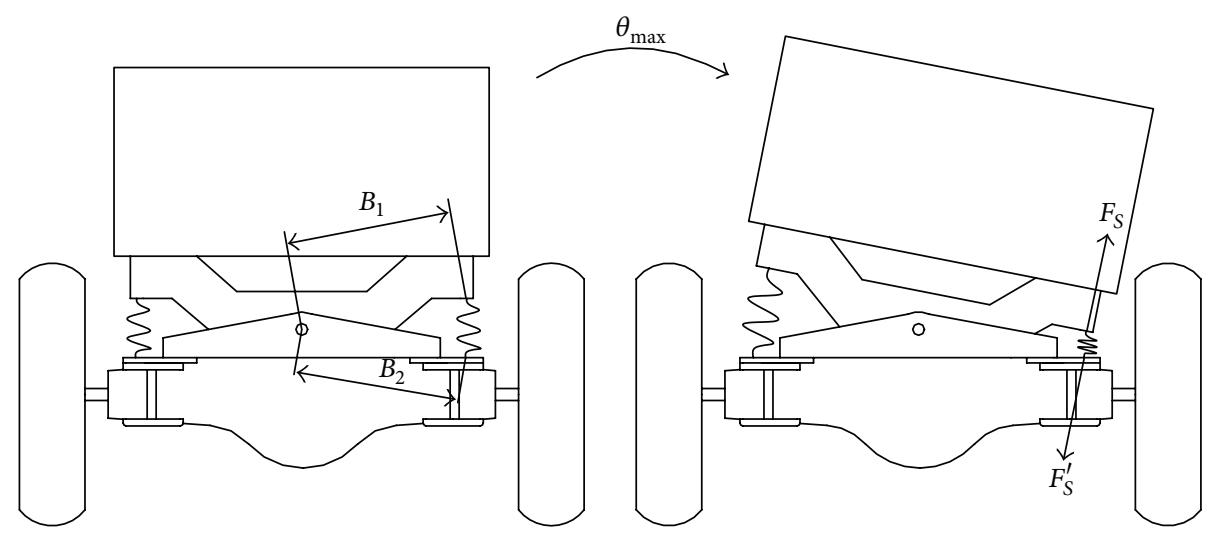

Figure 4: The sketch of the contact force between the swing bridge and the rear axle.

3.1. Side-Slip Angle. Lateral wheel slip is defined as (11). The side-slip angles of the tires in our model can be calculated using (12). Hence,

$$
\begin{aligned}
\tan \alpha_{i} & =-\frac{v_{y_{i}}}{v_{x i}} \\
\alpha_{1} & =-\arctan \frac{v_{y} \cos \delta-v_{x} \sin \delta+l_{f} r}{v_{x} \cos \delta+B r / 2} \\
\alpha_{2} & =-\arctan \frac{v_{y} \cos \delta-v_{x} \sin \delta+l_{f} r}{v_{x} \cos \delta-B r / 2} \\
\alpha_{3} & =-\arctan \frac{v_{y}-l_{r} r}{v_{x}+B r / 2} \\
\alpha_{4} & =-\arctan \frac{v_{y}-l_{r} r}{v_{x}-B r / 2} .
\end{aligned}
$$

\subsection{Normal Force. Consider}

$$
F_{z i}=-\left[K_{v i}\left(d_{i}-s_{i}\right)+C_{v i}\left(\dot{d}_{i}-\dot{s}_{i}\right)\right],
$$

where $d_{i}$ is each displacement of the four tires in the $z$ direction of the global coordinate system, which can be calculated as follows:

$$
\begin{aligned}
& d_{1}=z-l_{1} \theta-l_{3} \psi \\
& d_{2}=z+l_{2} \theta-l_{4} \psi \\
& d_{3}=z+l_{r} \psi-\frac{B}{2} \theta^{\prime} \\
& d_{4}=z+l_{r} \psi+\frac{B}{2} \theta^{\prime},
\end{aligned}
$$

where $s_{i}$ is the vertical displacement of the $i$ th tire caused by obstacles.

The ASVs may pass over various shapes of obstacles in actual conditions, but we cannot consider every obstacle in the theoretical analysis. In order to facilitate the establishment of a mathematical model of obstacles, regularly shaped obstacles have been chosen, such as triangular, circular, and square-shaped obstacles.

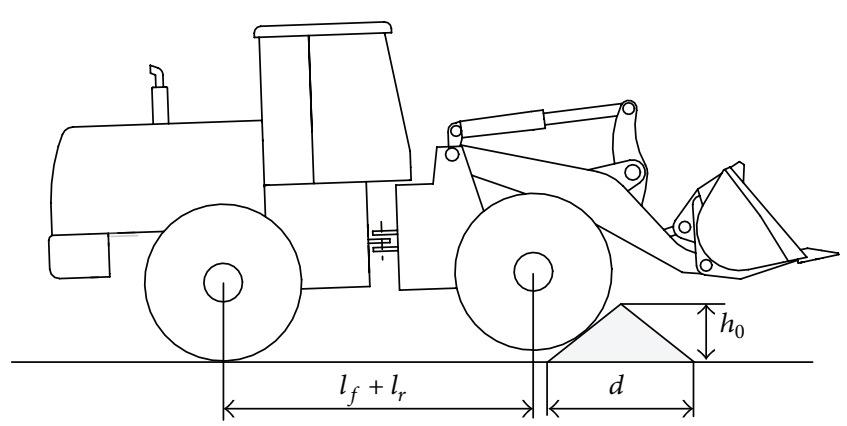

FIGURE 5: The sketch of the ASV passing over obstacle.

Figure 5 shows that the wheel loader passes over a triangular obstacle. As for the vehicle symmetry, this paper only analyzes roll stability of the ASVs when the left wheels pass over obstacles. $s_{i}$ can be written as follows.

The right front wheel $s_{1}=0$.

The left front wheel

$$
s_{2}= \begin{cases}0 & \left(0 \leq t<t_{0}\right) \\ -a\left(t-t_{0}\right) & \left(t_{0} \leq t<t_{0}+t_{d 1}\right) \\ a\left(t-t_{0}-t_{d 1}\right)+b & \left(t_{0}+t_{d 1} \leq t<t_{0}+t_{d}\right) \\ 0 & \left(t \geq t_{0}+t_{d}\right) .\end{cases}
$$

The right rear wheel $s_{3}=0$.

The left rear wheel

$s_{4}$

$$
= \begin{cases}0 & \left(0 \leq t<t_{0}+t_{l}\right) \\ -a\left(t-t_{0}-t_{l}\right) & \left(t_{0}+t_{l} \leq t<t_{0}+t_{l}+t_{d 1}\right) \\ a\left(t-t_{0}-t_{l}-t_{d 1}\right)+b & \left(t_{0}+t_{l}+t_{d 1} \leq t<t_{0}+t_{l}+t_{d}\right) \\ 0 & \left(t \geq t_{0}+t_{l}+t_{d}\right),\end{cases}
$$

where $t_{d 1}=d / 2 v, t_{d}=d / v$, and $t_{l}=\left(l_{r}+l_{f}\right) / v$.

In the solution process, $d_{i}-s_{i}>0$ indicates that the $i$ th wheel lifts off the ground; set $K_{v i}=0, C_{v i}=0$, and $K_{\alpha i}=0$. 


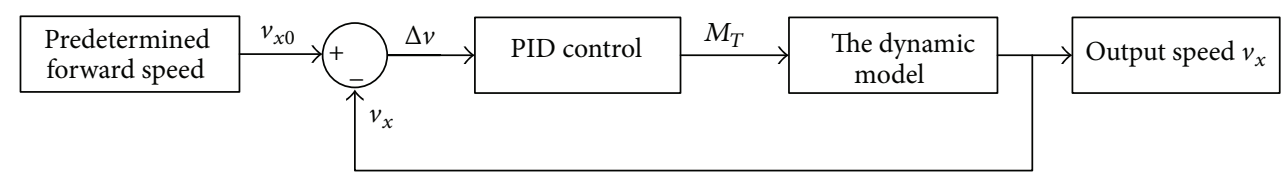

Figure 6: The block diagram of the speed control.

\subsection{Longitudinal Force. Consider}

$$
F_{x}= \begin{cases}K_{x} \lambda, & |\lambda| \leq \lambda_{\text {critical }} \\ -\operatorname{sign}(\lambda)\left(F_{x 1}-F_{x 2}\right), & |\lambda|>\lambda_{\text {critical }}\end{cases}
$$

where $\lambda_{\text {critical }}$ is the critical longitudinal slip which is used to determine whether tires are sliding or not:

$$
\lambda_{\text {critical }}=\left|\frac{\mu F_{z}}{2 K_{x}}\right| \text {. }
$$

The friction coefficient, depending on static friction $\left(\mu_{s}\right)$, sliding friction $\left(\mu_{d}\right)$, longitudinal slip ratio $(\lambda)$, and side-slip angle $(\alpha)$, is defined as

$$
\mu=\mu_{s}-\left(\mu_{s}-\mu_{d}\right) \sqrt{\lambda^{2}+\tan ^{2} \alpha}
$$

$F_{x 1}$ and $F_{x 2}$ can be calculated as follows:

$$
\begin{aligned}
& F_{x 1}=\mu\left|F_{z}\right| \\
& F_{x 2}=\left|\frac{\left(\mu F_{z}\right)^{2}}{\left(4 \lambda K_{x}\right)}\right| .
\end{aligned}
$$

$\lambda$, the longitudinal slip ratio, is calculated as follows:

$$
\lambda_{i}= \begin{cases}\frac{R_{i} \omega_{i}-v_{x i}}{R_{i} \omega_{i}} & \text { (acceleration: } \left.R_{i} \omega_{i} \geq v_{x i}\right) \\ \frac{R_{i} \omega_{i}-v_{x i}}{v_{x i}} & \text { (braking: } \left.R_{i} \omega_{i}<v_{x i}\right)\end{cases}
$$

The dynamics of each individual wheel are given by

$$
\dot{\omega}_{i}=\frac{M_{T i}-R_{i} F_{x i}}{I_{w}} \quad(i=1,2,3,4)
$$

where $M_{T i}$ is driving/braking torque and $I_{w}$ is rotational inertia of wheel.

This paper focuses on the effects of active braking on roll instability of the ASVs. The time derivative of the $i$ th wheel longitudinal slip in braking process is given by

$$
\dot{\lambda}_{i}=\frac{R_{i} \dot{\omega}_{i}}{v_{x i}}-\left(1+\lambda_{i}\right) \frac{\dot{v}_{x i}}{v_{x i}} .
$$

Substituting (22) into (23) gives

$$
\dot{\lambda}_{i}=-\frac{R_{i}^{2}}{I_{w} v_{x i}} F_{x i}-\left(1+\lambda_{i}\right) \frac{\dot{v}_{x i}}{v_{x i}}-\frac{R_{i}}{I_{w} v_{x i}} M_{T i} .
$$

\subsection{Lateral Force. Consider}

$$
F_{y}= \begin{cases}-\mu\left|F_{z}\right|\left(1-H^{3}\right) \operatorname{sign}(\alpha), & |\alpha| \leq \alpha_{\text {critical }} \\ -\mu\left|F_{z}\right| \operatorname{sign}(\alpha), & |\alpha|>\alpha_{\text {critical }}\end{cases}
$$

where $\alpha_{\text {critical }}$ is critical lateral slip, which is used to judge whether tires are sliding or not, $\alpha_{\text {critical }}=\arctan \left(3 \mu F_{z} / K_{\alpha}\right)$, and $H=1-\left(K_{\alpha}|\tan \alpha|\right) /\left(3 \mu\left|F_{z}\right|\right)$.

\section{The Model Solution}

The established dynamic model encompasses the driving/ braking torque $M_{T}$ and the steering torque $M_{Z}$. When the vehicle is in the process of driving and steering, the driving torque and the steering torque constantly change with the outside input. If the exact value of the real-time change is desired, it is necessary to establish the model of power transmission (engine, hydraulic torque converter, gearbox, and transfer case) and the model of hydraulic steering system, which will make the whole analysis very complex. In theoretical analysis, the forward speed and the steering angle of the vehicle can be controlled by a classical ProportionalIntegral-Derivative control (PID control).

Figure 6 is the block diagram of the speed control in the process of model solution. The difference $\Delta v$ between the predetermined speed $v_{x 0}$ and the output $v_{x}$ of the model is rendered by a PID to be the control on the driving/braking torque $M_{T}$. By adjusting $M_{T}$ in real time, the forward speed $v_{x}$ in the dynamic equation (1) is ensured to amount to the set value $v_{x 0}$.

The steering angle control is the same as the speed control. The difference $\Delta \delta$ between the predetermined steering angle and the output of the model is rendered by a PID to be the control on the steering torque $M_{Z}$. By adjusting $M_{Z}$ in real time, the steering angle $\delta$ in the dynamic equation (8) is guaranteed to track the set value $\delta_{0}$.

With the calculation methods of the driving torque, the steering torque, and the tire forces, the equation can be written as a MATLAB program. Figure 7 presents the block diagram of the dynamic model solution. It shows the whole calculation process of the dynamic model. Before calculation, the model parameters and the solution parameters need to be set first. The input of the model mainly includes the steering angle, the forward speed, and the road parameters.

\section{Experimental Verification of the Dynamic Model}

This paper studies the roll stability of the ASVs. Conducting experiments on real vehicles is risky. Moreover, real ASVs 


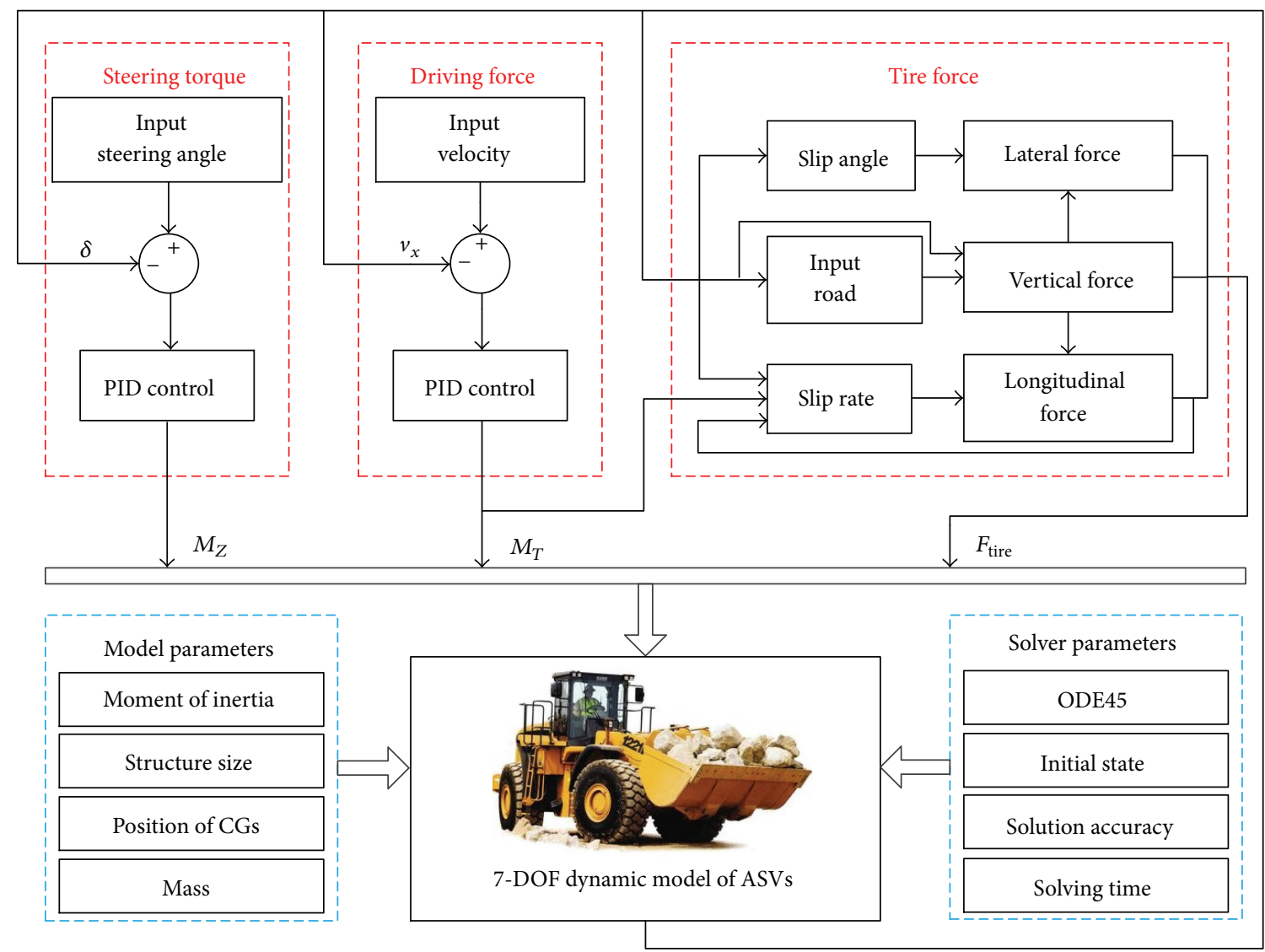

FIGURE 7: The block diagram of the dynamic model solution.

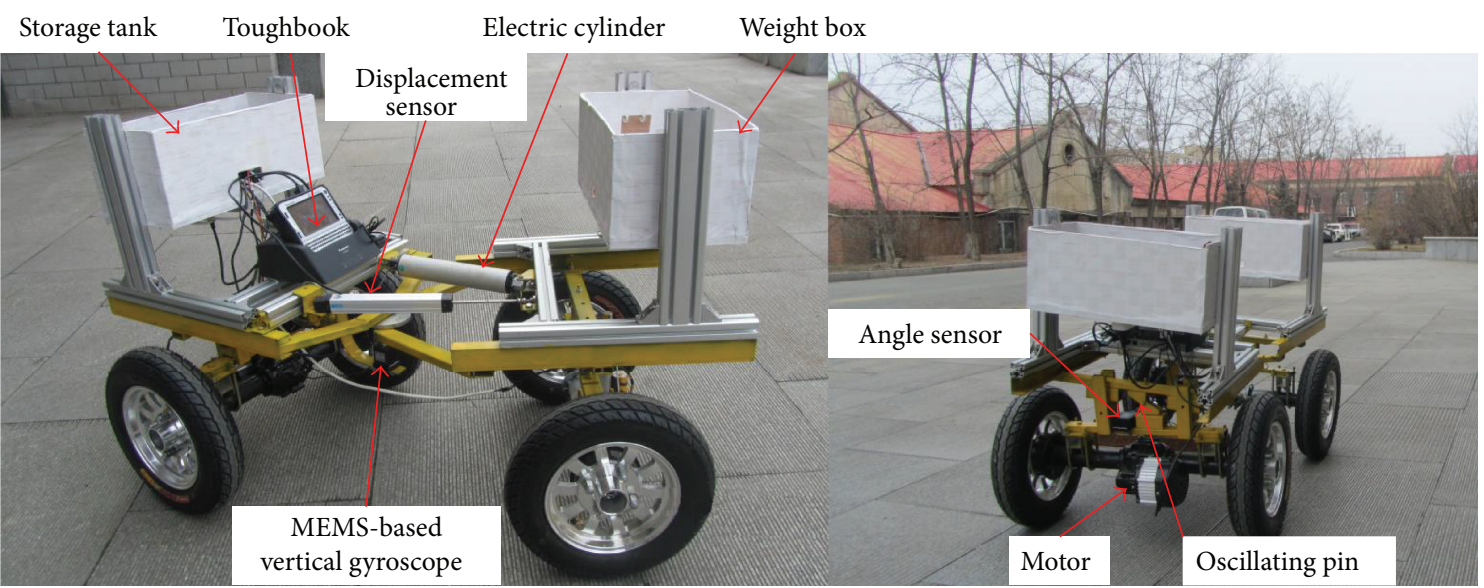

FIGURE 8: The experimental prototype of the ASV.

are difficult to modify. For these reasons, we designed an experiment prototype (scaled ASV) that has the main features of the ASVs to verify the mathematical model.

5.1. Introduction to the Experiment Prototype. The experiment prototype has the following features:

(1) The prototype is mainly composed of three parts: the front body, the rear body, and the rear axle. The front body is jointed with the rear body by a steering pin. The swing bridge is linked to the rear axle by an oscillating pin, as shown in Figure 8, and the upper part of the swing bridge has a fixed connection with the rear body.

(2) The experimental prototype adopts a DC speed regulating motor to drive the two rear wheels. An electric cylinder installed between the front body and the 
TABLE 1: Parameters of the scaled ASV and the ZL50 wheel loader.

\begin{tabular}{|c|c|c|c|}
\hline \multirow{2}{*}{ Parameters } & \multirow{2}{*}{ Meanings and units } & \multicolumn{2}{|c|}{ Value } \\
\hline & & Scaled ASV & ZL50 wheel loader \\
\hline$m$ & Total mass of ASV (kg) & 86.5 & 16747.4 \\
\hline$m_{1}$ & Front body mass (kg) & 43.8 & 6979.8 \\
\hline$m_{2}$ & Rear body mass (kg) & 26.1 & 8896.0 \\
\hline$m_{3}$ & Rear axle mass (kg) & 16.5 & 871.6 \\
\hline$J_{x x 1} / J_{y y 1} / J_{z z 1}$ & Moment of inertia of front body $\left(\mathrm{kg} \cdot \mathrm{m}^{2}\right)$ & $5.31 / 14.2 / 13.2$ & $5248 / 32310 / 32977$ \\
\hline$J_{x x 2} / J_{y y 2} / J_{z z 2}$ & Moment of inertia of rear body $\left(\mathrm{kg} \cdot \mathrm{m}^{2}\right)$ & $3.51 / 6.10 / 4.20$ & $5842 / 14982 / 13228$ \\
\hline$J_{x x 3} / J_{y y 3} / J_{z z 3}$ & Moment of inertia of rear axle $\left(\mathrm{kg} \cdot \mathrm{m}^{2}\right)$ & $1.71 / 3.13 / 3.85$ & $1428 / 1238 / 1431.0$ \\
\hline$W_{1}\left(X_{1}, Y_{1}, Z_{1}\right)$ & CG of front body (m) & $(0.477,0,0.111)$ & $(1.80,0.03,0)$ \\
\hline$W_{2}\left(X_{2}, Y_{2}, Z_{2}\right)$ & CG of rear body (m) & $(-0.328,0,0.270)$ & $(-1.86,0.06,0.61)$ \\
\hline$W_{3}\left(X_{3}, Y_{3}, Z_{3}\right)$ & CG of rear axle (m) & $(-0.391,0,-0.167)$ & $(-1.67,0,-0.41)$ \\
\hline$B$ & Tread $(\mathrm{m})$ & 0.70 & 2.30 \\
\hline$B_{2}$ & Distance between the oscillating pin and the limit position $(\mathrm{m})$ & 0.12 & 0.47 \\
\hline$l_{f}$ & Distance between the steering pin and the front axle (m) & 0.53 & 1.55 \\
\hline$l_{r}$ & Distance between the steering pin and the rear axle (m) & 0.40 & 1.67 \\
\hline$R_{t}$ & Tire radius $(\mathrm{m})$ & 0.21 & 0.87 \\
\hline$h$ & Distance between the oscillating pin and the rear axle (m) & 0.18 & 0.32 \\
\hline$K_{x}$ & Longitudinal stiffness of tire $(\mathrm{N} / \mathrm{m})$ & $1.76 \times 10^{5}$ & $9.7 \times 10^{6}$ \\
\hline$K_{\alpha}$ & Cornering stiffness of tire (N/deg) & $8.16 \times 10^{4}$ & $4.8 \times 10^{6}$ \\
\hline$K_{v}$ & Vertical stiffness of tire $(\mathrm{N} / \mathrm{m})$ & $5.1 \times 10^{4}$ & $2.9 \times 10^{6}$ \\
\hline$C_{v}$ & Vertical damping of tire $(\mathrm{N} \cdot \mathrm{s} / \mathrm{m})$ & $3.3 \times 10^{3}$ & $4.27 \times 10^{5}$ \\
\hline$I_{w}$ & Moment of inertia of wheel $\left(\mathrm{kg} \cdot \mathrm{m}^{2}\right)$ & 0.1 & 117.4 \\
\hline$K_{S}$ & Contact stiffness $(\mathrm{N} / \mathrm{m})$ & $1 \times 10^{8}$ & $1 \times 10^{8}$ \\
\hline$C_{S}$ & Contact damping $(\mathrm{N} \cdot \mathrm{s} / \mathrm{m})$ & $1 \times 10^{4}$ & $1 \times 10^{4}$ \\
\hline$\theta_{\operatorname{Max}}$ & Maximum angle of swing bridge (deg) & 15 & 15 \\
\hline
\end{tabular}

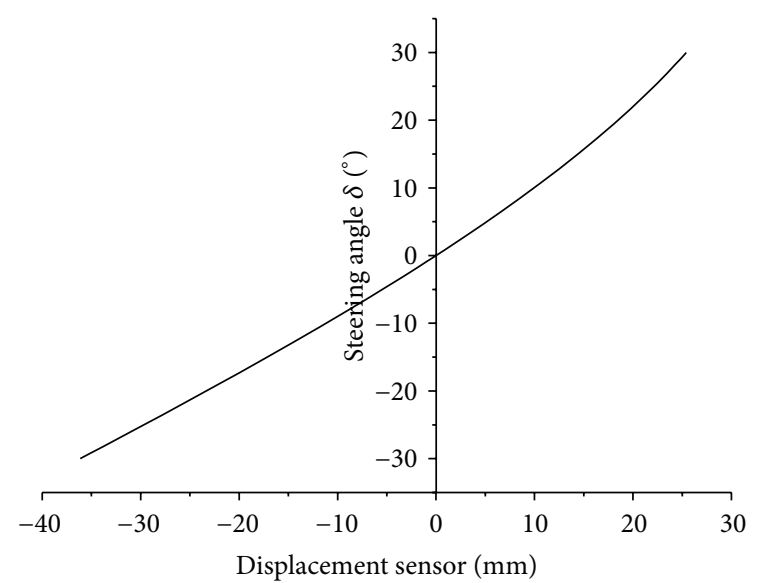

FIGURE 9: The corresponding relation of displacement sensor and steering angle.

rear body is used to steer. The articulated steering angle is measured by a displacement sensor (WYL100). The correspondence relationship between the displacement sensor and the steering angle is shown in Figure 9.

(3) The experimental prototype contains the necessary sensors to measure vehicular motions. A MEMSbased vertical gyroscope VG440CA-200 (Crossbow
Technology, Inc., Milpitas, CA, USA) is installed at the intersection point of the articulated steering pin axis and the oscillating pin axis to provide real-time vehicle kinematics data (velocities, accelerations, and angles). The angle sensor (XW-TS1130) installed on the rear axle is used to measure the roll angle of this axle. The NI data acquisition card (USB-6211) is taken as the acquisition system. A Panasonic Toughbook U1 CF-VEBU11AU (Panasonic Co., Ltd., Osaka, Japan) is installed at the rear frame to collect data and to control forward speed and articulated angle. Table 1 shows the dimensions and specifications of the scaled ASV. Note that the parameters, such as moment of inertia and center of gravity position, are obtained from the three-dimensional model of the ASVs built in software CATIA (Dassault Systèmes SA, France).

5.2. Experiments and Results Analysis. To confirm the relevant simulation conclusions, we conducted experiments under two experimental operating conditions, namely, (1) turning on flat roads and (2) passing over obstacle during turning. The inputs of the steering angle are shown in Figure 10. The base and the height of the triangle obstacle are $0.3 \mathrm{~m}$ and $0.1 \mathrm{~m}$, respectively. The shape and size of the obstacle and the process of passing over obstacle are shown in Figure 11. In addition, the parameters of static friction $\mu_{s}$ and sliding friction $\mu_{d}$ are set as 0.6 and 0.4 , respectively. 
TABLE 2: Twelve kinds of vehicle motions in condition 1.

\begin{tabular}{lcccccccccccc}
\hline & 1 & 2 & 3 & 4 & 5 & 6 & 7 & 8 & 9 & 10 & 11 & 12 \\
\hline Steering angle $\left(^{\circ}\right)$ & 20 & 20 & 20 & 20 & 25 & 25 & 25 & 25 & 30 & 30 & 30 & 30 \\
Velocity $(\mathrm{m} / \mathrm{s})$ & 6 & 7 & 8 & 9 & 6 & 6.5 & 7 & 7.5 & 5 & 6 & 6.5 & 7 \\
\hline
\end{tabular}

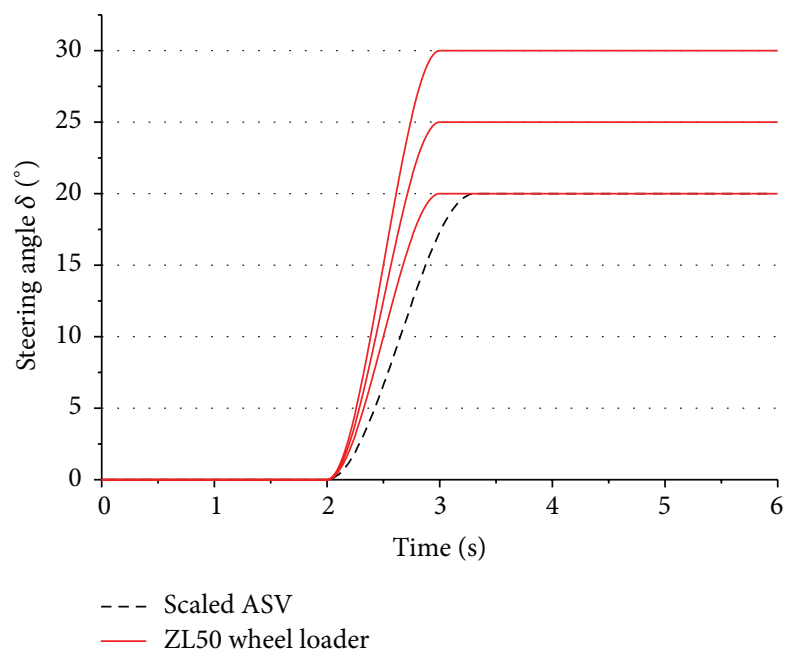

FIGURE 10: The inputs of the steering angle.
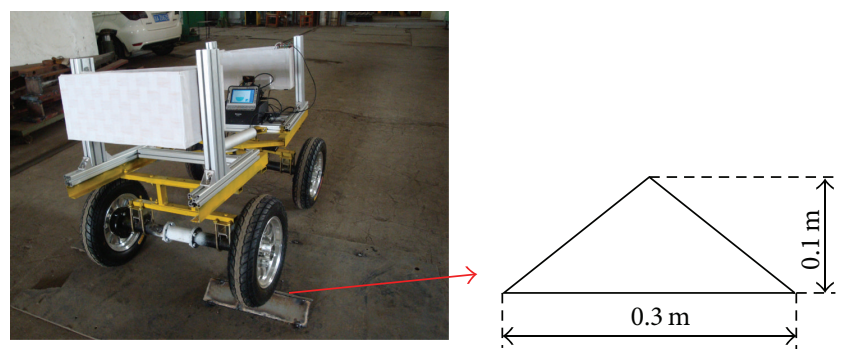

FIGURE 11: The shape and size of the obstacle and the process of passing over obstacle.

5.2.1. Turning on Flat Road. Figure 12 illustrates the motions of the scaled ASV when turning on a flat road. The results indicate good agreement between yaw rate and lateral acceleration of the model and the test data. Yaw rate and lateral acceleration are simultaneously increased with increasing forward speed from $1 \mathrm{~m} / \mathrm{s}$ to $2 \mathrm{~m} / \mathrm{s}$, which is entirely consistent with the actual situation. While some deviations are also evident, as it is observed in Figure 12(c), the lateral acceleration of the vehicle model at time range $2.5 \mathrm{~s}-6 \mathrm{~s}$ is above the measured data, which may partially be attributed to the constant speed in simulation. In the dynamic model, the vehicle advances at a constant speed, and the velocity is maintained at $l \mathrm{~m} / \mathrm{s}$ $(2 \mathrm{~m} / \mathrm{s})$ when turning. In practice, however, maintaining vehicle velocity at $l \mathrm{~m} / \mathrm{s}(2 \mathrm{~m} / \mathrm{s})$ is difficult because speed often decreases when turning. Therefore, the yaw rate and the lateral acceleration obtained by the simulation are slightly higher than those obtained by the test during cornering.

Given that the vehicle turned on a flat road at low speed, the pitch and vertical motion were not obvious and were therefore not described in this work.
5.2.2. Passing over Obstacle during Turning. Figure 13 summarizes the results of both the field experiments and the simulations of scaled ASV motion when turning on uneven ground. The results show good agreement between the model and the test data. The vehicle has been in the turning state before coming into contact with the obstacle, such that the initial values of the lateral acceleration and yaw rate are not equal to zero. The ASV tilts to the right, and the roll angular velocity increases rapidly when the left front wheel comes into contact with the obstacle. Meanwhile, the roll angle positively increases, while the pitch angle negatively increases. The left rear wheel begins to come into contact with the obstacle at $3 \mathrm{~s}$, such that the roll angle of the rear axle and the pitch angle positively increase. The lateral acceleration of the vehicle will drastically change when the wheels touch and leave the obstacle. Vehicle forward speed will change when passing over the obstacle, and the yaw rate will vary with speed. The scaled ASV will return to the steady state after passing over the obstacle.

\section{Study on Roll Instability Mechanism of the ASVs}

The dynamic model is applied to analyze the lateral stability of the ZL50 wheel loader (produced by XCMG Construction Machinery Co., Ltd., Xuzhou, China). Table 1 lists the parameters of the ZL50 wheel loader. To determine the roll instability mechanism of the ASVs, the following four conditions have been studied: (1) quick turn on flat road; (2) passing over obstacles when driving in a straight line; (3) passing over obstacles when turning; and (4) passing over obstacles on a sloping road.

Here, we choose the lateral transfer ratio (LTR) as stability index to indicate the vehicle rollover propensity, which is defined as

$$
\operatorname{LTR}=\left|\frac{F_{z R}-F_{z L}}{F_{z R}+F_{z L}}\right|=\left|\frac{\left(F_{z 1}+F_{z 3}\right)-\left(F_{z 2}+F_{z 4}\right)}{F_{z 1}+F_{z 3}+F_{z 2}+F_{z 4}}\right| .
$$

The LTR varies from 0 to 1 , where 0 refers to equal vertical forces on both sides of the vehicle (zero roll) and 1 refers to either the left or the right vehicle tires losing contact with the ground [11].

6.1. Stability Analysis of Condition 1: Quick Turn on Flat Road. To determine the instability mechanism of the ASVs in condition 1, we analyzed the vehicle motions corresponding to 12 kinds of parameters, which are listed in Table 2. According to the analysis results, vehicle instability will occur (the value of LTR reaches 1, as shown in Figure 14(a)) when the steering angle is $20^{\circ}, 25^{\circ}$, and $30^{\circ}$ and the forward speed exceeds $8 \mathrm{~m} / \mathrm{s}, 7 \mathrm{~m} / \mathrm{s}$, and $6.5 \mathrm{~m} / \mathrm{s}$, respectively. 


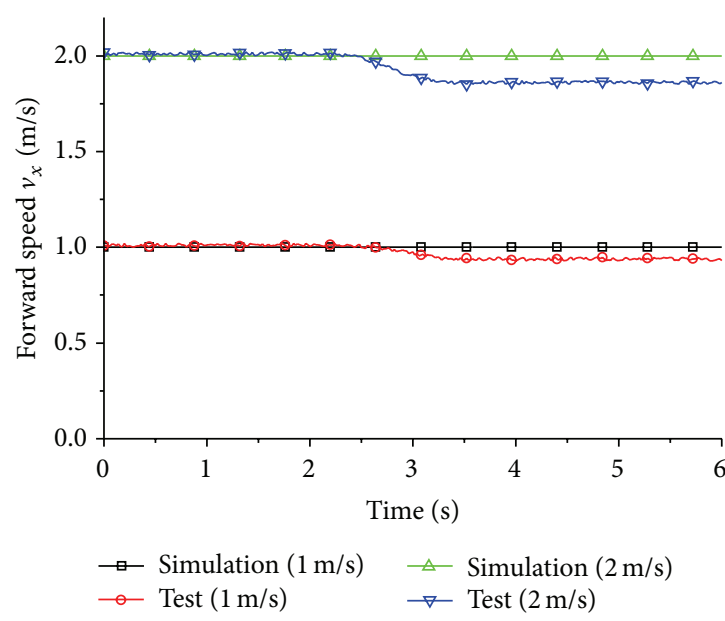

(a)

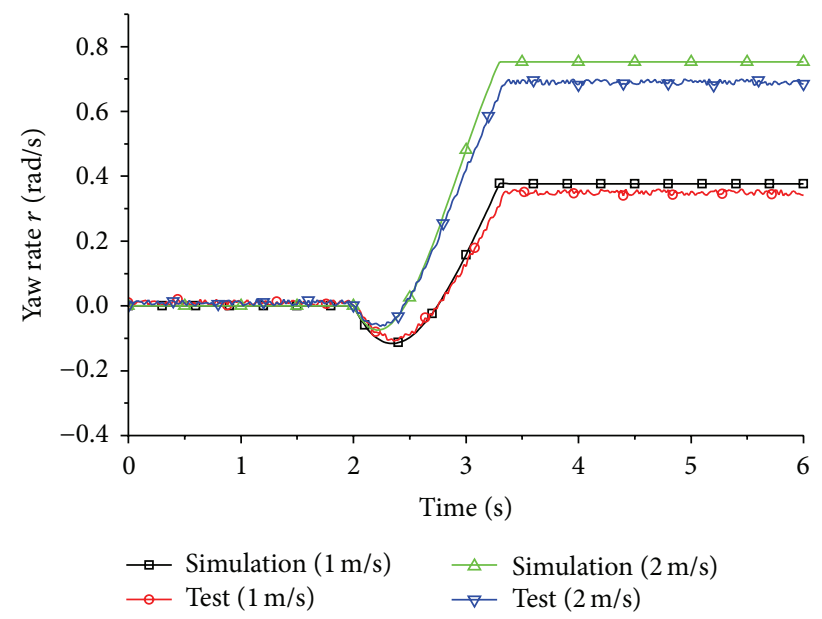

(b)

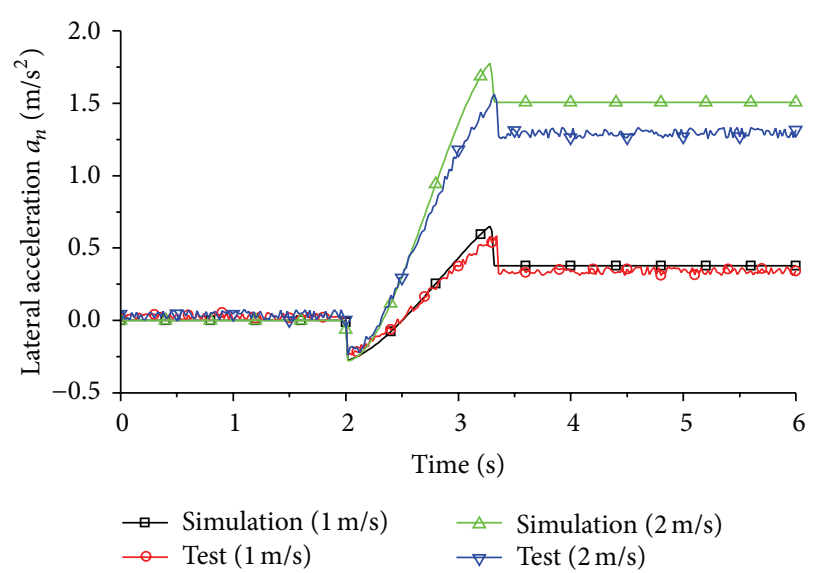

(c)

FIGURE 12: Comparisons of simulation results with test data when the ASV turns on flat road.

Figures 14(b), 14(c), and 14(d) show the vehicle motions corresponding to critical rollover instability. The lateral acceleration reaches $5 \mathrm{~m} / \mathrm{s}^{2}$, the roll angular velocity is close to $0.6 \mathrm{rad} / \mathrm{s}$, and the yaw rate varies from $0.6 \mathrm{rad} / \mathrm{s}$ to $0.8 \mathrm{rad} / \mathrm{s}$ when the critical instability occurs in condition 1 . Roll instability is mainly caused by the excessive lateral acceleration or the excessive yaw rate when the wheel loader turns on a flat road. The value of the yaw rate is not constant when the wheel loader loses stability under different conditions. Thus, the yaw rate cannot be used as the stability index in condition 1 . We therefore take lateral acceleration as the stability index when the ASVs turn on a flat road. In this analysis, the wheel loader will lose stability when the lateral acceleration reaches $5 \mathrm{~m} / \mathrm{s}^{2}$.

6.2. Stability Analysis of Condition 2: Passing over Obstacles When Driving in Straight Line. The shapes of obstacles are triangular and circular, as shown in Figure 15. Each shape has two different sizes, such that obstacles are of four types: (1) $s_{t}=0.3 \mathrm{~m}, d_{t}=0.8 \mathrm{~m}$; (2) $s_{t}=0.4 \mathrm{~m}, d_{t}=0.8 \mathrm{~m}$; (3) $s_{c}=$ $0.25 \mathrm{~m}, d_{c}=0.8 \mathrm{~m}$; and (4) $s_{c}=0.3 \mathrm{~m}, d_{c}=0.8 \mathrm{~m}$.

By analyzing the movement of the wheel loader as it passes over the four obstacles at different speeds, we found that the wheel loader will lose stability (the value of LTR reaches 1) when the forward speed reaches $7 \mathrm{~m} / \mathrm{s}, 6 \mathrm{~m} / \mathrm{s}$, $6.4 \mathrm{~m} / \mathrm{s}$, and $5 \mathrm{~m} / \mathrm{s}$. By comparing the wheel loader motion parameters, such as lateral acceleration, roll angular velocity, and vertical acceleration, the roll angular velocity is found to be close to $3 \mathrm{rad} / \mathrm{s}$ under the four critical instability conditions, as shown in Figure 16. Therefore, the roll stability of ASVs can be expressed by the roll angular velocity when the ASVs pass over obstacles in a straight line. In this analysis, the wheel loader is in a stable state when the roll angular velocity is less than $3 \mathrm{rad} / \mathrm{s}$; otherwise, the wheel loader will lose stability.

6.3. Stability Analysis of Condition 3: Passing over Obstacles When Turning. The stability of the wheel loader has been analyzed under two conditions: (1) turning on flat road and (2) passing over obstacles when driving in a straight line. The results showed that the stability under condition 1 mainly depends on the lateral acceleration, whereas that under condition 2 mainly depends on the roll angular velocity. We then analyze the condition when the wheel loader passes over obstacles when turning and study the relationship between the lateral acceleration and the roll angular velocity when 


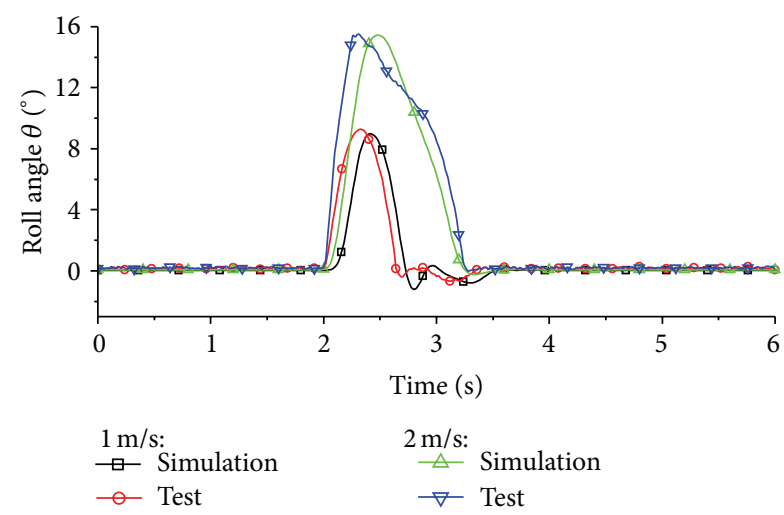

(a)

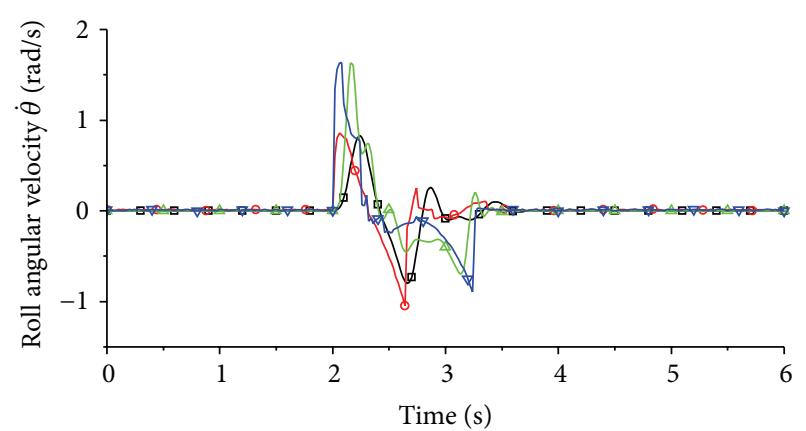

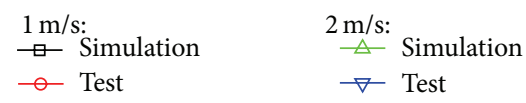

(c)

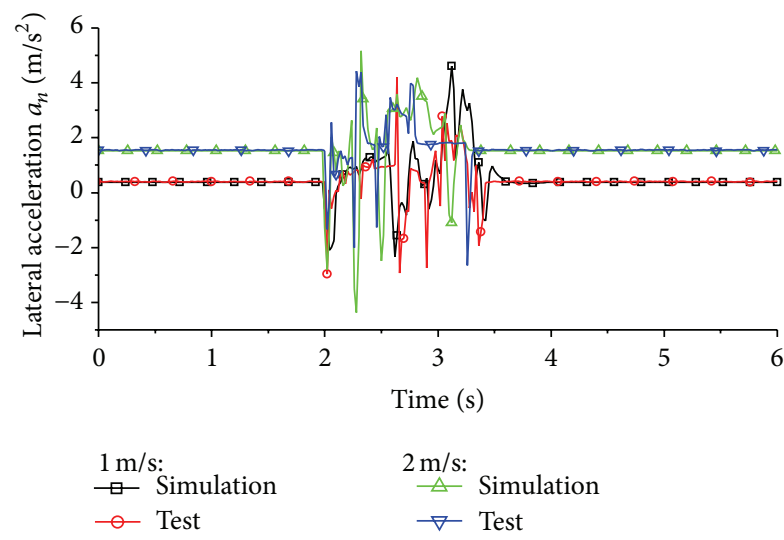

(e)

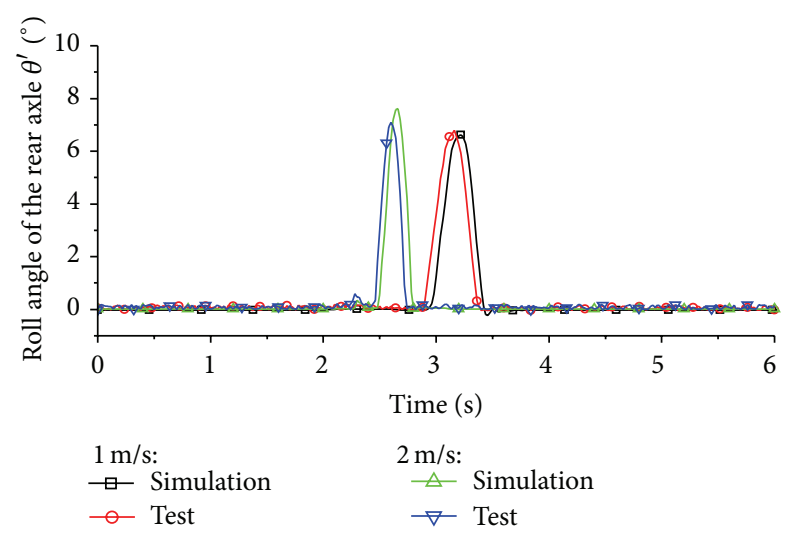

(b)

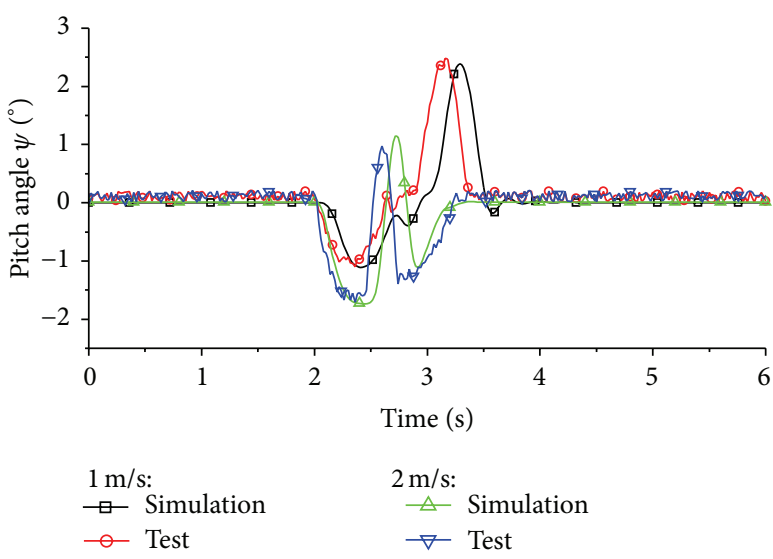

(d)

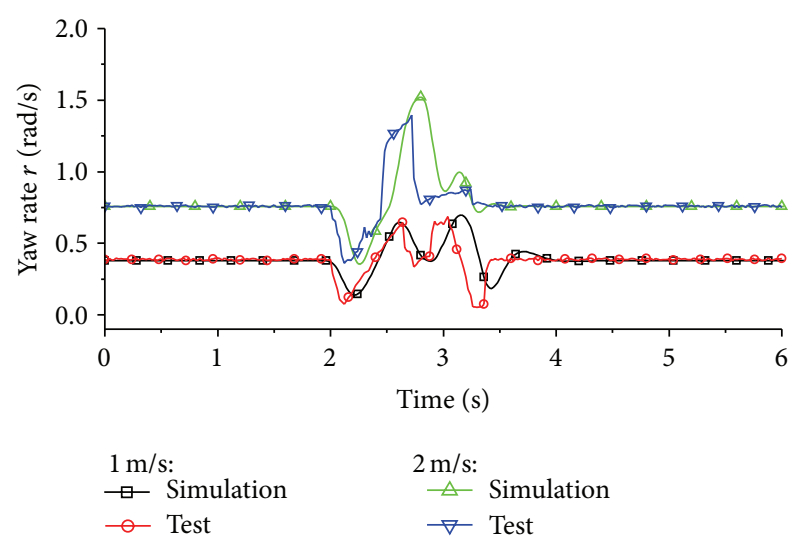

(f)

FIGURE 13: Comparisons of simulation results with test data when the ASV passes over obstacles.

the ASVs lose stability. The lateral acceleration values are set as $1 \mathrm{~m} / \mathrm{s}^{2}, 1.5 \mathrm{~m} / \mathrm{s}^{2}, 2 \mathrm{~m} / \mathrm{s}^{2}, 2.5 \mathrm{~m} / \mathrm{s}^{2}, 3 \mathrm{~m} / \mathrm{s}^{2}, 3.5 \mathrm{~m} / \mathrm{s}^{2}$, and $4 \mathrm{~m} / \mathrm{s}^{2}$ by adjusting the steering angle and the forward speed. The wheel loader loses stability by adjusting the size of the obstacles when the vehicle is running with one of the above lateral acceleration values. We obtain the roll angular velocity corresponding to critical rollover instability in each of the above lateral acceleration values.

Taking the lateral acceleration as the $x$-axis and taking the critical roll angular velocity as the $y$-axis, we can draw the relationship between the lateral acceleration and the roll angular velocity, as shown in Figure 17. When the wheel loader is traveling in a straight line, and the lateral acceleration is equal to 0 , instability will occur once the roll angular velocity exceeds $3 \mathrm{rad} / \mathrm{s}$, which is consistent with the result in Section 6.2. The roll angular velocity that caused instability will decrease as lateral acceleration gradually increases. The wheel loader will directly lose stability when the lateral acceleration increases to $5 \mathrm{~m} / \mathrm{s}^{2}$, which is consistent with the result in Section 6.1. In addition, the roll angular velocity rapidly 


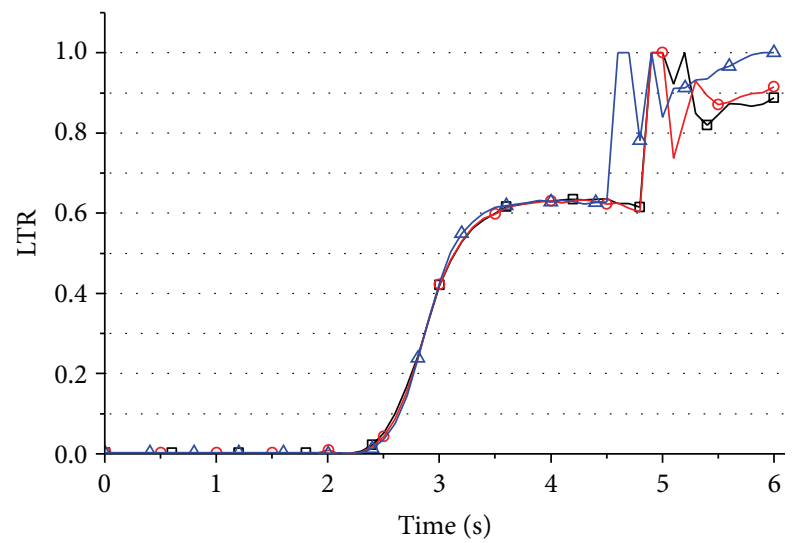

$\square$ Steering angle $20^{\circ}$, velocity $8 \mathrm{~m} / \mathrm{s}$

$\bigcirc$ Steering angle $25^{\circ}$, velocity $7 \mathrm{~m} / \mathrm{s}$

$\triangle$ Steering angle $30^{\circ}$, velocity $6.5 \mathrm{~m} / \mathrm{s}$

(a)

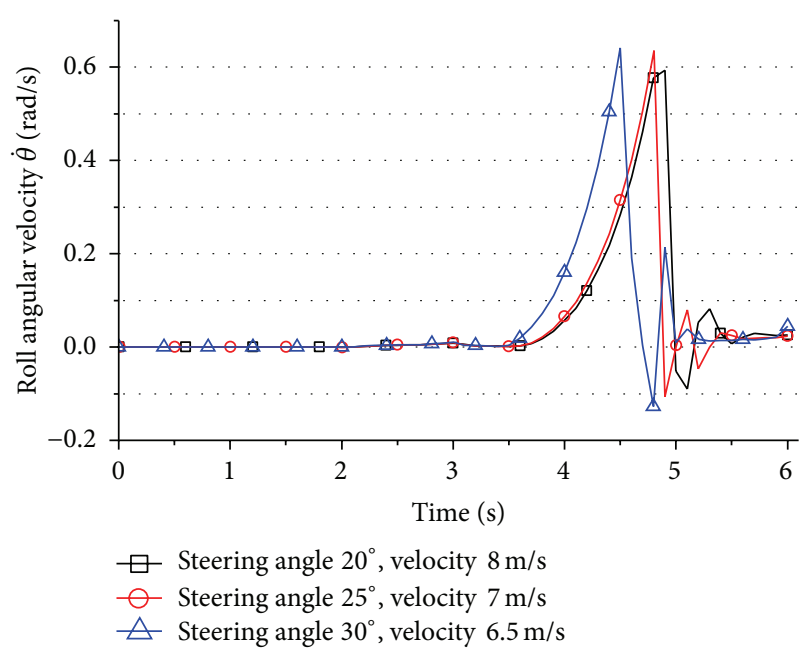

(c)

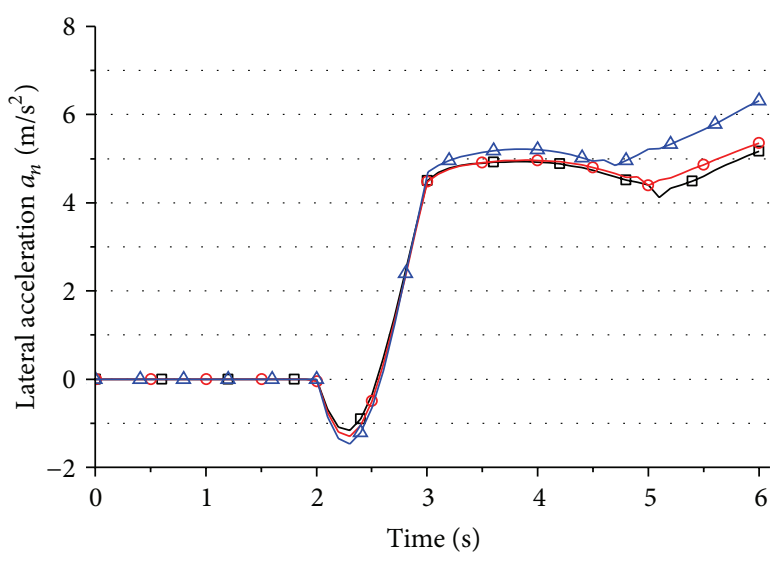

$\square$ Steering angle $20^{\circ}$, velocity $8 \mathrm{~m} / \mathrm{s}$

$\bigcirc$ Steering angle $25^{\circ}$, velocity $7 \mathrm{~m} / \mathrm{s}$

$\triangle$ Steering angle $30^{\circ}$, velocity $6.5 \mathrm{~m} / \mathrm{s}$

(b)

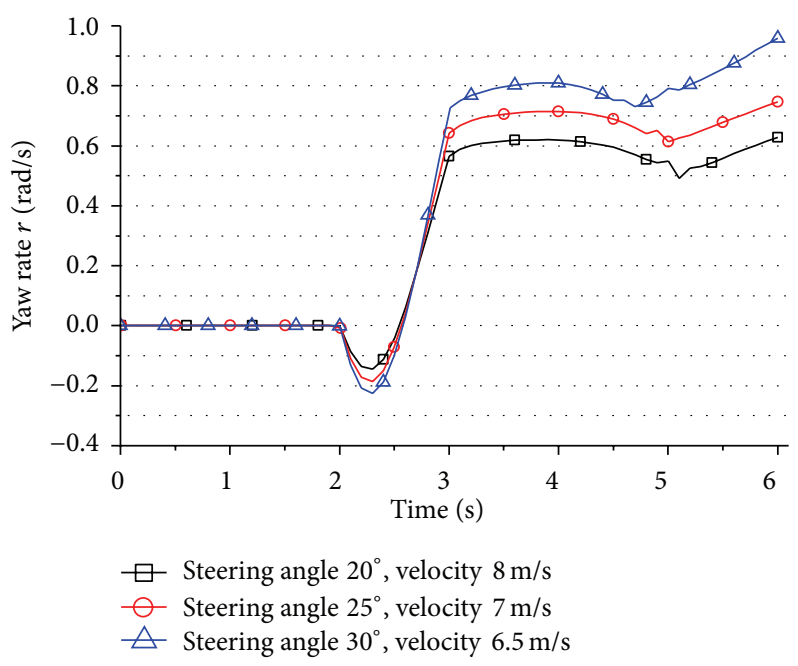

(d)

FIGURE 14: Movement states of the wheel loader under the critical instability in condition 1.
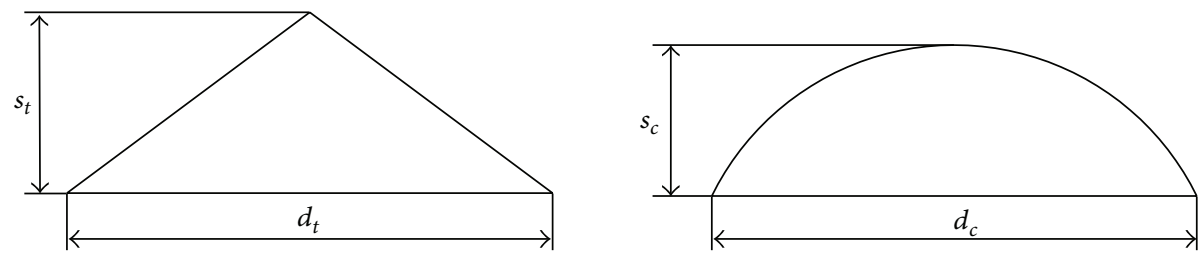

FIGURE 15: The shapes of the obstacles.

decreases when the lateral acceleration exceeds $4 \mathrm{~m} / \mathrm{s}^{2}$, such that instability of the vehicle is mainly determined by the lateral acceleration at this time.

6.4. Stability Analysis of Condition 4: Passing over Obstacles on Slopes. When the wheel loader is traveling on a sloping road, as shown in Figure 18, the gravitational acceleration $g$ generates subgravitational acceleration $a_{1}=g \sin \phi$ in the $y$-direction. This condition indicates that the lateral acceleration of the vehicle is not equal to 0 and the roll angular velocity that caused instability will change when the vehicle travels on a sloping road.

In the above analysis, we need to consider the factor of the slope angle $\phi$ and improve the dynamic model established in 


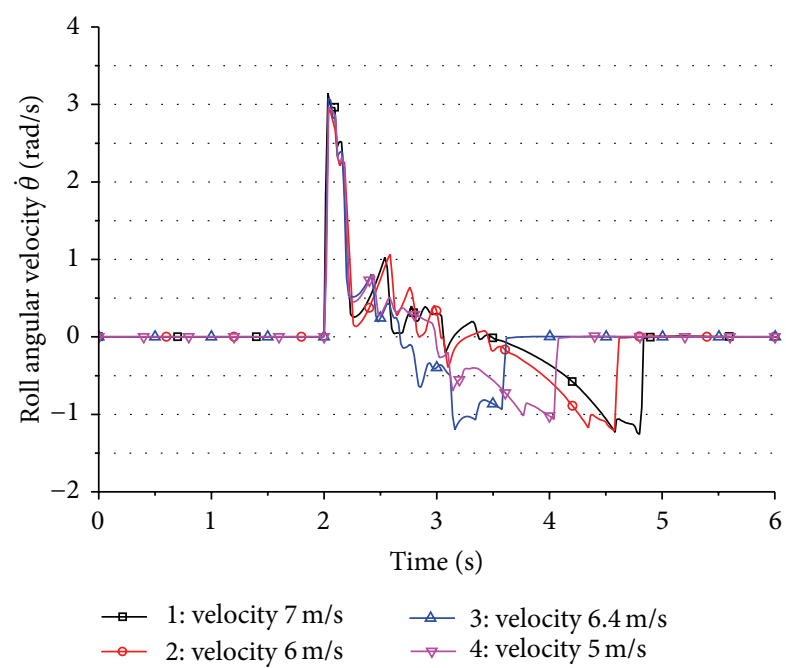

FIGURE 16: The roll angular velocity corresponding to the critical instability in condition 2.

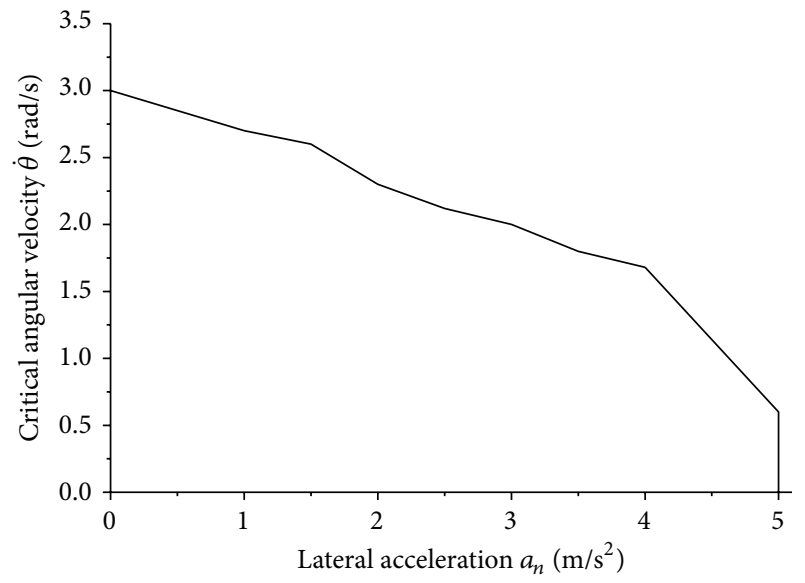

FIGURE 17: The relationship between the lateral acceleration and the critical angular velocity under instability.

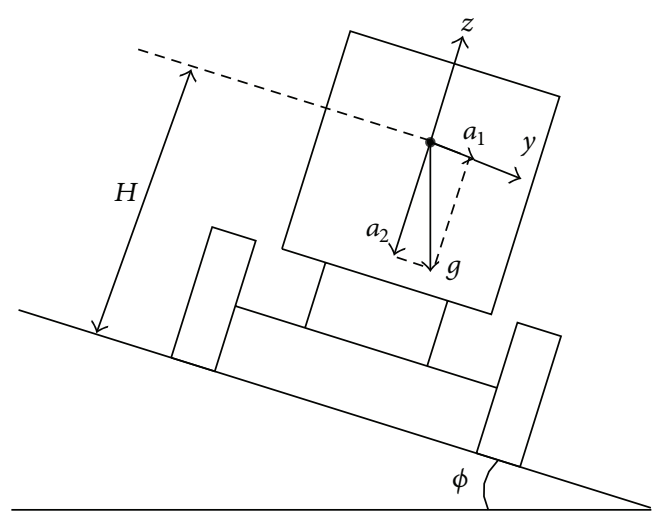

FIGURE 18: The schematic of the wheel loader traveling on slope road.
Section 2. Here, we analyze the condition in which the wheel loader is traveling in a straight line on a sloping road. The improved dynamic model is shown as follows:

(1) Vehicle motion along the $x$-axis direction:

$$
m\left(\dot{v}_{x}+\dot{z} \dot{\psi}\right)=\sum_{i=1}^{4}\left(F_{x i}+\frac{M_{T i}}{R_{t}}\right) .
$$

(2) Vehicle motion along the $y$-axis direction:

$$
m\left(\dot{v}_{y}-\dot{z} \dot{\theta}\right)=\sum_{i=1}^{4} F_{y i}-\sum_{i=1}^{3} G_{i} \sin \phi .
$$

(3) Vehicle motion along the $z$-axis direction:

$$
m\left(\ddot{z}+v_{y} \dot{\theta}-v_{x} \dot{\psi}\right)=\sum_{i=1}^{4} F_{z i}-\sum_{i=1}^{3} G_{i} \cos \left(\theta_{i}+\phi\right),
$$

where $\theta_{1}=\theta_{2}=\theta, \theta_{3}=\theta^{\prime}$.

(4) The roll motion of the front body and the rear body:

$$
\begin{aligned}
\left(J_{x x 1}+J_{x x 2}\right) \ddot{\theta}= & \sum_{i=1}^{2}\left[F_{y i}\left(R_{t}+h\right)+G_{i} Z_{i} \sin (\theta+\phi)\right] \\
& +\frac{\left(F_{z 2}-F_{z 1}\right) B}{2}-F_{S} B_{1} .
\end{aligned}
$$

(5) The roll motion of the rear axle:

$$
\begin{aligned}
J_{x x 3} \ddot{\theta}^{\prime}= & \left(F_{y 3}+F_{y 4}\right)\left(R_{t}+h\right)+\frac{\left(F_{z 4}-F_{z 3}\right) B}{2} \\
& +G_{3} Z_{3} \sin \left(\theta^{\prime}+\phi\right)+F_{S}^{\prime} B_{2} .
\end{aligned}
$$

(6) Vehicle pitch motion around the $y$-axis:

$$
\begin{aligned}
\left(J_{y y 1}\right. & \left.+J_{y y 2}+J_{y y 3}\right) \ddot{\psi} \\
= & -\left(F_{z 1}+F_{z 2}\right) l_{f}+\left(F_{z 3}+F_{z 4}\right) l_{r}+\sum_{i=1}^{3} G_{i} X_{i} \cos \phi \\
& -\sum_{i=1}^{4}\left(F_{x i}+\frac{M_{T i}}{R_{t}}\right)\left(R_{t}+h\right)+\sum_{i=1}^{3} m_{i} \dot{v}_{x} Z_{i} .
\end{aligned}
$$

Slope angles are set as $2.5^{\circ}, 5^{\circ}, 7.5^{\circ}, 10^{\circ}, 12.5^{\circ}, 15^{\circ}$, and $17.5^{\circ}$. The shape of the obstacle is triangular, the base $d_{t}=0.8 \mathrm{~m}$, and the height $s_{t}=0.3 \mathrm{~m}$. The wheel loader loses stability by adjusting the forward speed when the vehicle is traveling on a road with one of the above slope angles. We can obtain the roll angular velocity corresponding to the critical rollover instability in each of the above slope angles.

Taking the slope angle as the $x$-axis and the critical roll angular velocity as the $y$-axis, we can draw the relationship between the slope angle and the roll angular velocity, as shown in Figure 19. The slope angle and the roll angular velocity exhibit an exponential relationship. When the slope angle is equal to 0 , instability will occur once the roll angular velocity exceeds $3 \mathrm{rad} / \mathrm{s}$, which is consistent with the result in Section 6.2. A larger slope angle results in a smaller roll angular velocity corresponding to critical instability. 


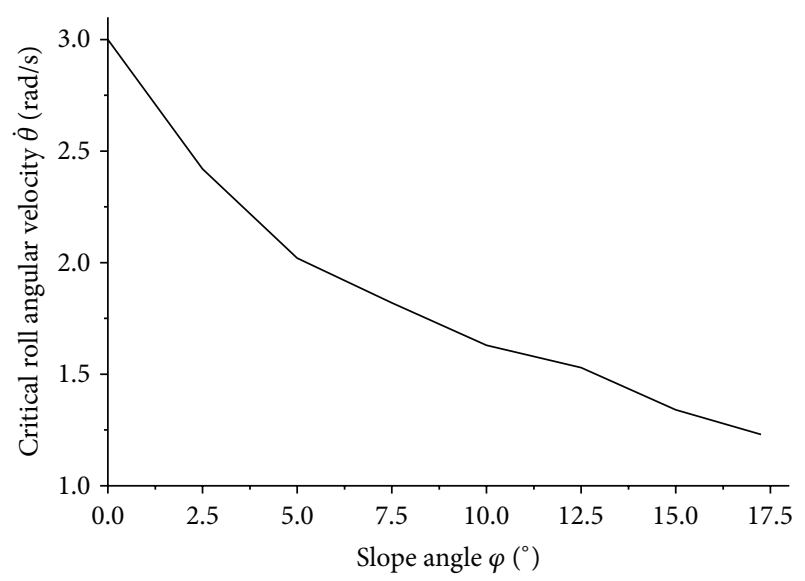

FIGURE 19: The relationship between the slope angle and the critical angular velocity under instability.

\section{Stability Index of the ASVs}

In summarizing the previous analysis, the following results can be obtained:

(1) Stability mainly depends on the lateral acceleration $a_{n}$ when the wheel loader is turning on a flat road. The wheel loader is in a stable state when the lateral acceleration is less than $5 \mathrm{~m} / \mathrm{s}^{2}$; otherwise, the vehicle will lose stability.

(2) Stability mainly depends on the roll angular velocity $\dot{\theta}$ when the wheel loader is passing over obstacles in a straight line. The wheel loader is in a stable state when the roll angular velocity is less than $3 \mathrm{rad} / \mathrm{s}$; otherwise, the vehicle will lose stability.

(3) Stability depends on both the lateral acceleration $a_{n}$ and the roll angular velocity $\dot{\theta}$ when the wheel loader is passing over obstacles while turning. The roll angular velocity that leads to the ASVs rollover will decrease with the increase of lateral acceleration.

(4) Critical roll angular velocity is affected by the slope angle when the wheel loader is traveling on a sloping road. A larger slope angle results in smaller roll angular velocity corresponding to instability.

According to the above results, we can conclude that the roll stability of ASVs is determined by the roll angular speed, lateral acceleration, and slope angle. The relationship between the roll angular velocity and the lateral acceleration can be expressed as in Figure 17. The relationship between the roll angular velocity and the slope angle can be expressed as in Figure 19. After obtaining the relationship among the roll angular speed, lateral acceleration, and slope angle, the SI of the ASVs can be uniformly expressed with the roll angular velocity, as defined in (33). SI > 0 indicates that the ASVs are in a stable state. A greater SI value indicates better ASVs stability. $S I=0$ indicates that the ASVs are in a critical state

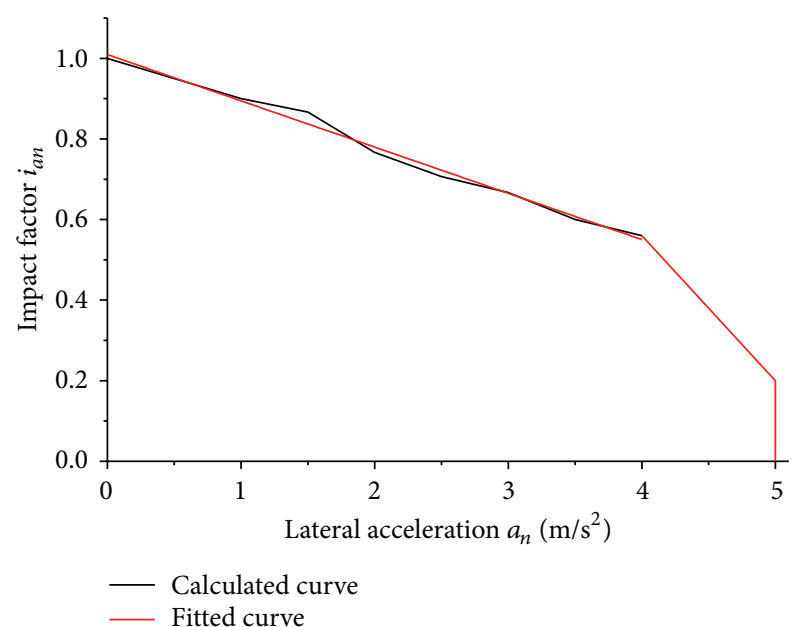

FIGURE 20: The influence coefficient of the lateral acceleration on the rollover threshold.

of instability. SI < 0 indicates that the ASVs lose stability. A smaller SI indicates poorer stability. Hence,

$$
S I=1-\frac{\dot{\theta}}{\dot{\theta}_{\text {critical }} i_{a n} i_{\varphi}},
$$

where $\dot{\theta}_{\text {critical }}$ is the initial rollover threshold and $\dot{\theta}_{\text {critical }}=$ $3 \mathrm{rad} / \mathrm{s}$ in this study. $i_{a n}$ is the influence coefficient of the lateral acceleration on the rollover threshold, $0<i_{a n} \leq 1 . i_{\varphi}$ is the influence coefficient of the slope angle on the rollover threshold, $0<i_{\varphi} \leq 1$.

The influence coefficients of $i_{a n}$ and $i_{\varphi}$ can be calculated from Figures 17 and 19, respectively. We transform Figure 17 into the form shown in Figure 20 and linearly fit the curve in Figure 20. The expression of influence coefficient $i_{a n}$ can thus be obtained:

$$
i_{a n}= \begin{cases}-0.115 a_{n}+1.0 & a_{n} \leq 4 \mathrm{~m} / \mathrm{s}^{2} \\ -1.08 a_{n}+6.0 & 4 \mathrm{~m} / \mathrm{s}^{2}<a_{n} \leq 5 \mathrm{~m} / \mathrm{s}^{2} \\ \longrightarrow 0^{+} & a_{n}>5 \mathrm{~m} / \mathrm{s}^{2}\end{cases}
$$

In the same way, we transform Figure 19 into the form shown in Figure 21 and fit the curve in Figure 21. The expression of impact factor $i_{\varphi}$ can thus be obtained:

$$
i_{\varphi}=0.689 e^{-\phi / 8.9}+0.311
$$

\section{Conclusions}

In this study, a typical ASV, that is, a wheel loader, is selected as the research object. A dynamic ASV model is established and verified with experiments. On this basis, a detailed analysis of the dynamic rollover stability of a wheel loader is conducted. The main conclusions are as follows:

(1) According to the structure and the movement characteristics of the ASVs, a 7-DOF nonlinear dynamic 


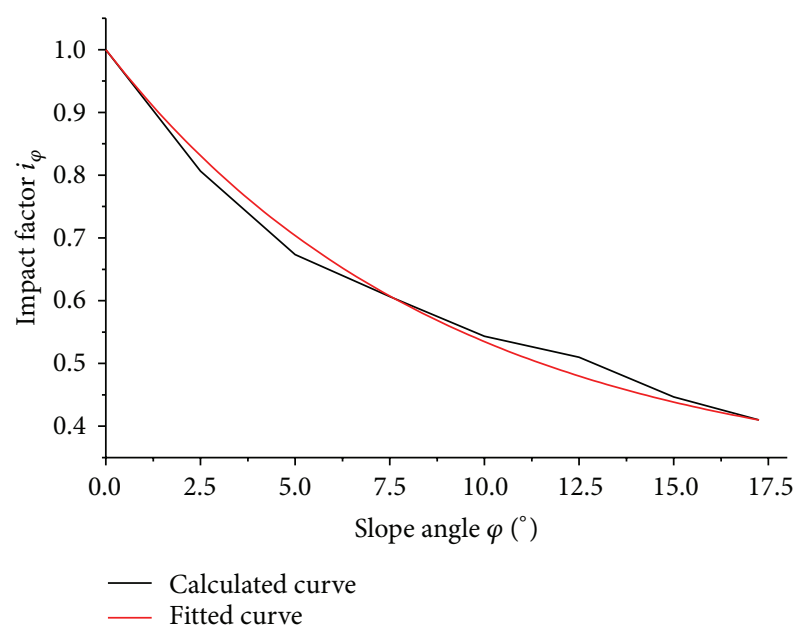

FIGURE 21: The influence coefficient of the slope angle on the rollover threshold.

model is established. This model considers articulated steering movement, swing bridge movement, and ground environmental parameters. To verify the reliability of the established model, a physical prototype of ASV is designed and fabricated. Experiments are conducted under two types of working conditions: turning on flat roads and passing over obstacle during turning. The experimental results mostly conform to the simulation results of the dynamic model, which indicates that the established dynamic model can truly reflect the movement characteristics of the ASVs.

(2) Detailed analysis of the rollover stability of a typical ASV, that is, a ZL50 wheel loader, is conducted under different conditions. The results show that (a) roll instability is mainly caused by excessive lateral acceleration when the ASV is turning on a flat road; (b) roll instability is mainly caused by the large angular velocity when the ASV passes over obstacles in a straight line; (c) roll stability is determined by both the angular velocity and the lateral acceleration when the ASV passes over obstacles during turning; and (d) the slope angle can decrease the ASV stability.

(3) In summarizing the various instability conditions, we can conclude that the roll instability of the ASVs is mainly caused by excessive lateral acceleration, large angular velocity, and oversized slope angle. Considering all the factors that cause instability, the $S I$ of the ASVs is presented. The SI indicates the ASVs stability by detecting whether the roll angular velocity exceeds the critical threshold. The SI considers information on roll angular velocity, lateral acceleration, and slope angle. This index can thus be used as early warning index for the ASVs.

\section{Competing Interests}

The authors declare that there is no conflict of interests regarding the publication of this paper.

\section{Acknowledgments}

This study was supported by the National Natural Science Foundation of China (Grants no. 51505177 and no. 51505176). The authors are also indebted to all members of the laboratory for helping to do experiments in the entire process.

\section{References}

[1] N. L. Azad, A. Khajepour, and J. Mcphee, "Robust state feedback stabilization of articulated steer vehicles," Vehicle System Dynamics, vol. 45, no. 3, pp. 249-275, 2007.

[2] Y. Yavin, "Modelling the motion of an underground mining vehicle," Mathematical and Computer Modelling, vol. 42, no. 910, pp. 1123-1130, 2005.

[3] P. I. Corke and P. Ridley, "Steering kinematics for a centerarticulated mobile robot," IEEE Transactions on Robotics and Automation, vol. 17, no. 2, pp. 215-218, 2001.

[4] B. J. Dragt, F. R. Camisani-Calzolari, and I. K. Craig, "Modelling the dynamics of a Load-Haul-Dump vehicle," in Proceedings of the 16th Triennial World Congress of International Federation of Automatic Control (IFAC '05), pp. 49-54, Prague, Czech Republic, July 2005.

[5] S. Rakheja and A. Piche, "Development of directional stability criteria for an early warning safety device," SAE Technical Paper 902265, 1990.

[6] R. D. Ervin, "Two active systems for enhancing dynamic stability in heavy truck operations," Tech. Rep. UMTRI-98-39, 1998.

[7] R. Strickland and H. McGee, "Evaluation of prototype automatic truck rollover warning systems," Tech. Rep. FHWA-RD97-124, Federal Highway Administration, Washington, DC, USA, 1998.

[8] A. Nalecz, Z. Lu, and K. d'Entremont, "An investigation into dynamic measures of vehicle rollover propensity," SAE Technical Paper 930831, 1993.

[9] J. Liu and P. D. Ayers, "Off-road vehicle rollover and field testing of stability index," Journal of Agricultural Safety and Health, vol. 5, no. 1, pp. 59-71, 1999.

[10] J. Liu and P. D. Ayers, "Application of a tractor stability index for protective structure deployment," Journal of Agricultural Safety and Health, vol. 4, no. 5, pp. 171-181, 1998.

[11] J. Preston-Thomas and J. H. Woodrooffe, "A feasibility study of a rollover warning device for heavy trucks," TP 10610E, Transport Canada, 1990.

[12] D. Odenthal, "Nonlinear steering and braking control for vehicle rollover avoidance," in Proceedings of the IEEE European Control Conference (ECC '99), pp. 598-603, Kalsruhe, Germany, September 1999.

[13] A. J. P. Miège and D. Cebon, "Active roll control of an experimental articulated vehicle," Proceedings of the Institution of Mechanical Engineers, Part D: Journal of Automobile Engineering, vol. 219, no. 6, pp. 791-806, 2005.

[14] S. Solmaz, M. Corless, and R. Shorten, "A methodology for the design of robust rollover prevention controllers for automotive vehicles with active steering," International Journal of Control, vol. 80, no. 11, pp. 1763-1779, 2007.

[15] H. B. Pacejka, Tire and Vehicle Dynamics, Society of Automotive Engineers, Warrendale, Pa, USA, 2002. 


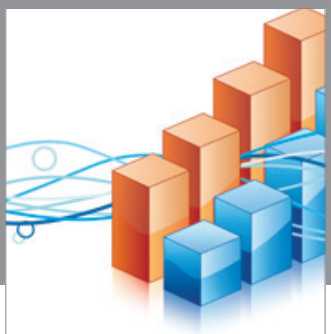

Advances in

Operations Research

vatem alat4

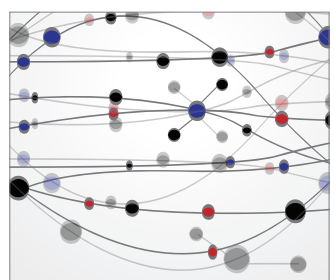

\section{The Scientific} World Journal
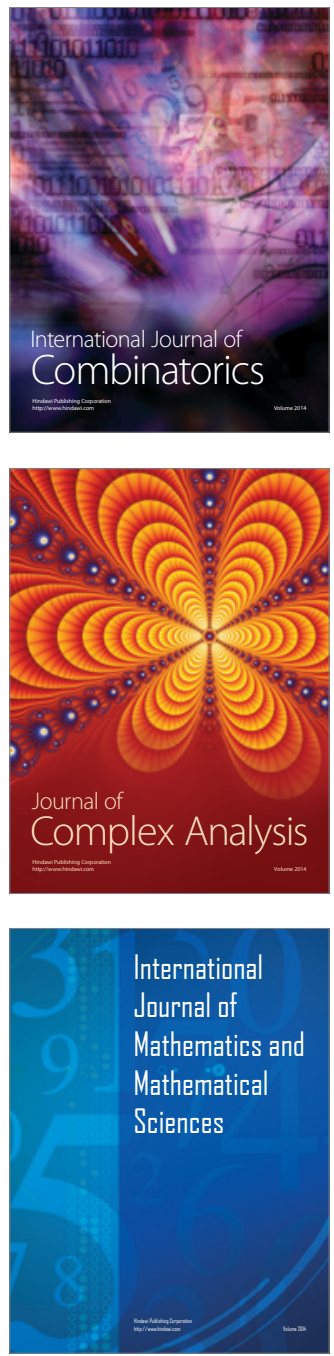
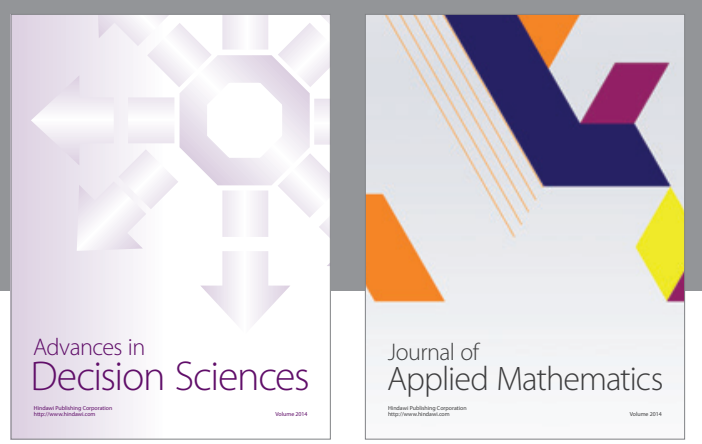

Algebra

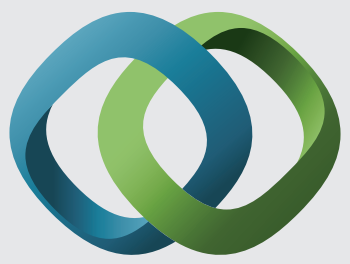

\section{Hindawi}

Submit your manuscripts at

http://www.hindawi.com
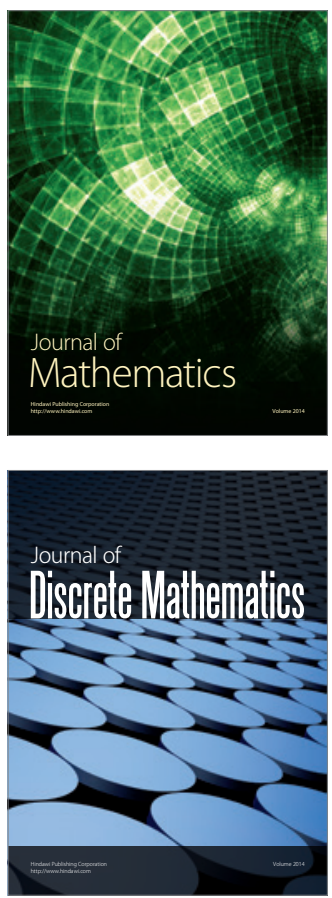

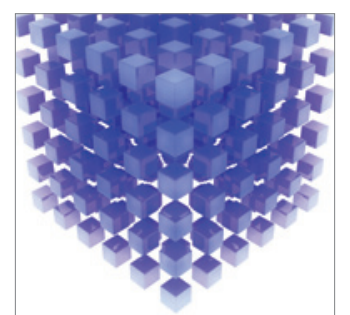

Mathematical Problems in Engineering
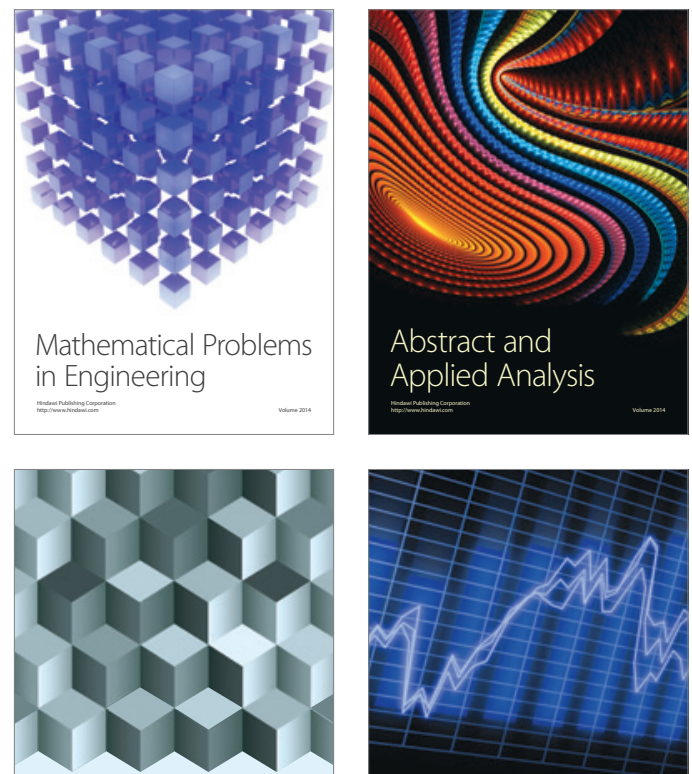

Journal of

Function Spaces

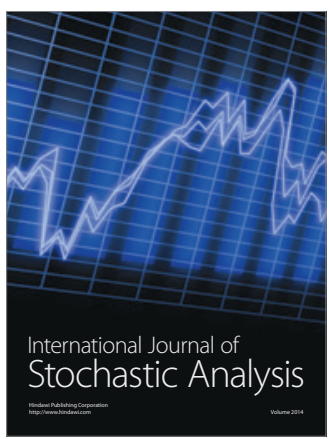

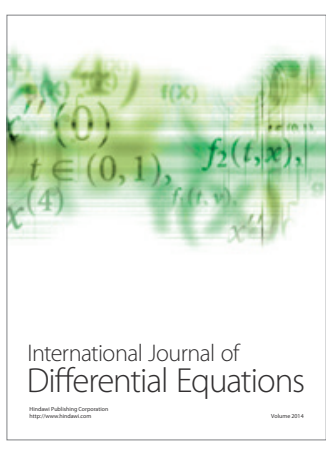
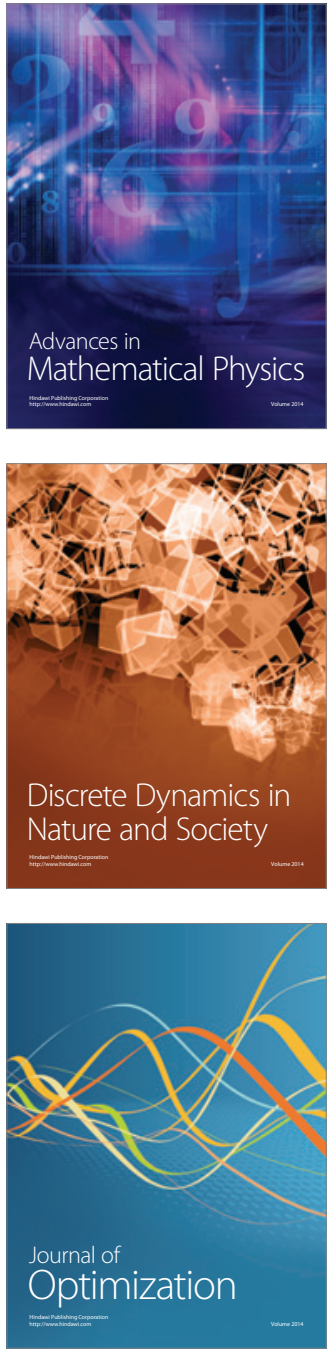